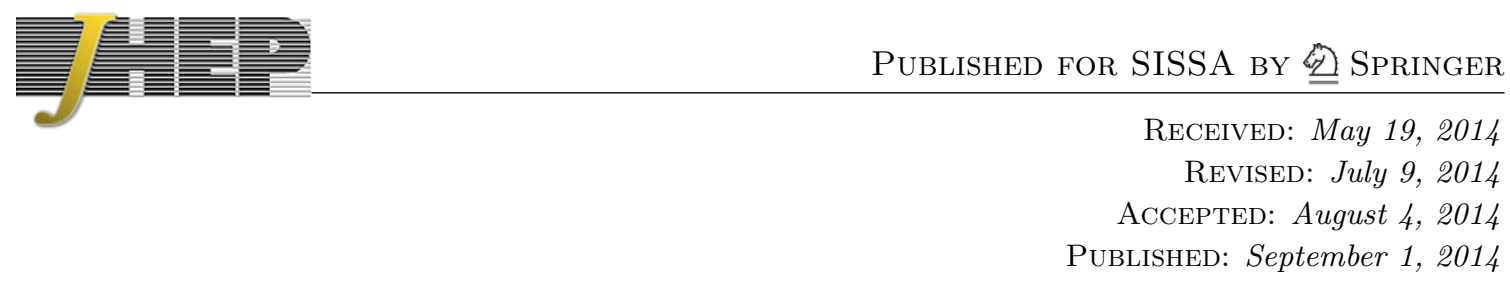

\title{
Resummed Higgs cross section at $\mathrm{N}^{3} \mathrm{LL}$
}

\author{
Marco Bonvini $^{a}$ and Simone Marzani ${ }^{b}$ \\ ${ }^{a}$ Deutsches Elektronen-Synchroton, DESY, \\ Notkestraße 85, D-22603 Hamburg, Germany \\ ${ }^{b}$ Institute for Particle Physics Phenomenology, Durham University, \\ South Road, Durham DH1 3LE, England \\ E-mail: marco.bonvini@desy.de, simone.marzani@durham.ac.uk
}

ABSTRACT: We present accurate predictions for the inclusive production of a Higgs boson in proton-proton collisions, via gluon-gluon fusion. Our calculation includes nextto-next-to-leading order (NNLO) corrections in perturbative QCD, as well as the resummation of threshold-enhanced contributions to next-to-next-to-next-to-leading logarithmic $\left(\mathrm{N}^{3} \mathrm{LL}\right)$ accuracy, with the inclusion of the recently-determined three-loop constant coefficient (sometimes referred to as $\mathrm{N}^{3} \mathrm{LL}^{\prime}$ accuracy).

Our result correctly accounts for finite top, bottom and charm masses at leading order (LO) and next-to-leading order (NLO), and includes top mass dependence at NNLO. At the resummed level the dependence on top, bottom and charm mass is accounted for at NLL, while only the top mass at NNLL. The all-order calculation is improved by a suitable choice of the soft terms, dictated by analyticity conditions and by the inclusion of subleading corrections of collinear origin, which improve the accuracy of the resummation away from the threshold region.

We present results for different collider energies and we study perturbative uncertainties by varying renormalization and factorization scales. We find that, at current LHC energies, the resummation corrects the NNLO result by as much as $20 \%$ at $\mu_{\mathrm{R}}=\mu_{\mathrm{F}}=m_{\mathrm{H}}$, while the correction is much smaller, $5.5 \%$, at $\mu_{\mathrm{R}}=\mu_{\mathrm{F}}=m_{\mathrm{H}} / 2$. While the central value of $\mathrm{NNLO}+\mathrm{N}^{3} \mathrm{LL}$ result depends very mildly on the scale choice, we argue that a more realiable estimate of the theoretical uncertainty is found if the perturbative scales are canonically varied about $m_{\mathrm{H}}$.

KeYwords: Higgs Physics, Resummation, QCD, Standard Model

ARXIV EPRINT: 1405.3654 


\section{Contents}

1 Introduction 1

2 Soft-gluon resummation $\quad 3$

2.1 Generalities 3

2.2 Large $N$ limit $-N$-soft 5

$\begin{array}{lll}2.3 & \text { Prescriptions for the Landau pole } & 7\end{array}$

$\begin{array}{lll}3 & \text { Soft terms and analyticity conditions } & 7\end{array}$

3.1 Functional form of the soft terms 8

$\begin{array}{llr}3.2 & \text { Altarelli-Parisi contributions } & 9\end{array}$

$\begin{array}{lll}3.3 & A \text {-soft }_{2} & 10\end{array}$

$3.4 \psi$-soft $_{2}-11$

4 Hadron-level results $\quad 11$

$\begin{array}{lll}4.1 \text { A study of the scale dependence } & 12\end{array}$

$\begin{array}{lll}4.2 & \text { Numerical results for different collider energies } & 17\end{array}$

$\begin{array}{llr}5 & \text { Conclusions } & 19\end{array}$

$\begin{array}{ll}\text { A Prescriptions and their implementations } & \mathbf{2 0}\end{array}$

A.1 Minimal Prescription 20

A.2 Borel Prescription 21

\section{Introduction}

The resummation of soft-gluon (or threshold) logarithms in QCD plays an important role in precision phenomenology at hadron colliders, and in particularly at the LHC. Examples include Higgs boson production in gluon fusion, e.g. [1], top-pair production, e.g. [2] and supersymmetric particles, e.g. [3]. Soft gluon resummation improves the accuracy of the predicted cross section, leading, for instance, to a reduced scale dependence.

This is particularly important in the case of Higgs production in gluon-gluon fusion. QCD corrections are fully known up to next-to-next-to-leading order (NNLO) accuracy [4$15]$ and $\mathrm{N}^{3} \mathrm{LO}$ calculations are underway [16-34]. Very recently, the first term in the soft expansion of the full $\mathrm{N}^{3} \mathrm{LO}$ cross section has been obtained [35]. The perturbative behavior of this series is very poor and thus logarithmically enhanced soft terms, predicted to all orders by soft-gluon resummation, provide a powerful tool to include (and check) higher order terms in the series.

Strictly speaking, soft-gluon resummation is needed when the partonic subprocess is close to threshold: being $M$ the mass of the tagged final state (the Higgs boson mass, for 
instance) and $\sqrt{\hat{s}}$ the center-of-mass energy of the partonic subsystem, in the limit $z=$ $M^{2} / \hat{s} \rightarrow 1$ the QCD perturbative expansion of the partonic cross section is unstable and the resummation of the entire series is necessary. Whether this is the case in the computation of the physical hadron-level cross section depends both on hadron-level kinematics and on the shape of parton distribution functions (PDFs), since the physical cross section is a convolution of the partonic cross section and PDFs.

In most cases, and particularly for inclusive observables at the LHC, the partonic region $z \rightarrow 1$ for which perturbativity is lost gives only a moderate, often negligible, contribution to the physical cross section [36]. In these cases, soft-gluon resummation is no longer needed; however, it might still be advisable. Indeed, an intermediate range of values of $z$ for which the soft terms approximate well the full partonic cross section usually exists [36, 37]; although in this region the series is behaving in a perturbative way, inclusion of higher order terms from soft-gluon resummation leads to a more accurate and stable prediction for the partonic cross section. When this intermediate range dominates the physical cross section, soft-gluon resummation provides then a powerful way of including (the dominant part of) higher order terms in the perturbative expansion.

The ability of all-order calculations to capture the region of intermediate $z$ strongly depends on the actual form of the soft terms that are being resummed [36, 37]. Indeed, while the soft limit determines the large- $z$ (or large- $N$, being $N$ the conjugate variable of $z$ upon Mellin transformation) behavior of the soft terms, it does not fix their functional form. Traditionally, the $N$-space resummation of the soft terms is organised in terms of powers of $\log N$ and constants. However, using analyticity arguments [37], we arrived at the conclusion that this choice is not optimal in several respects, chiefly because powers of $\log N$ exhibit a branch cut at finite $N$, in contrast to the pole structure of fixed-order coefficient functions. A form of the resummation that respects these analyticity properties is advisable, in particular if we aim to capture the dominant behavior in the region of intermediate $N$, i.e. intermediate $z$.

Following our previous studies $[37,38]$, we consider a functional form for the soft terms that respects the analyticity properties of fixed-order results. We improve on that work by implementing this formalism in an all-order resummation formula and by computing the Higgs production cross section at $\mathrm{NNLO}+\mathrm{N}^{3} \mathrm{LL}$, for different collider energies. Our result includes all the information from the $\mathrm{N}^{3} \mathrm{LO}$ soft-virtual calculation of ref. [35], and reproduces to order $\alpha_{s}^{3}$ the soft part of the $\mathrm{N}^{3} \mathrm{LO}$ approximate prediction of ref. [38]. We also study a different form of soft terms, which has the advantage of respecting the aforementioned analyticity conditions, while having, at the same time, a fast numerical implementation.

Finally, we note that a form of threshold resummation with the correct singularity structure at finite $N$ is a necessary step towards the construction of a double-resummed cross section in which threshold and high-energy (BFKL) logarithms are simultaneously accounted for to all orders. 


\section{Soft-gluon resummation}

\section{$2.1 \quad$ Generalities}

A physical (hadron-level) inclusive cross section at hadron colliders can be written in the factorized form

$$
\sigma\left(\tau, M^{2}\right)=\tau \sum_{i j} \int_{\tau}^{1} \frac{d z}{z} \mathscr{L}_{i j}\left(\frac{\tau}{z}, \mu_{\mathrm{F}}^{2}\right) \frac{1}{z} \hat{\sigma}_{i j}\left(z, M^{2}, \alpha_{s}\left(\mu_{\mathrm{R}}^{2}\right), \frac{M^{2}}{\mu_{\mathrm{F}}^{2}}, \frac{M^{2}}{\mu_{\mathrm{R}}^{2}}\right), \quad \tau=\frac{M^{2}}{s},
$$

where $\mathscr{L}_{i j}\left(z, \mu^{2}\right)$ is a parton luminosity

$$
\mathscr{L}_{i j}\left(z, \mu^{2}\right)=\int_{z}^{1} \frac{d x}{x} f_{i}\left(\frac{z}{x}, \mu^{2}\right) f_{j}\left(x, \mu^{2}\right)
$$

and $i, j$ run over all parton flavours. Without loss of generality, we can suppress the flavour indices and concentrate on the dominant channels for soft resummation ( $g g$ for Higgs). For ease of notation, we also suppress factorization scale $\mu_{\mathrm{F}}$ and renormalization scale $\mu_{\mathrm{R}}$ dependence. The partonic cross section $\hat{\sigma}$ is related to the so-called dimensionless coefficient function $C$ by

$$
\hat{\sigma}\left(z, M^{2}\right)=z \sigma_{0}\left(M^{2}\right) C\left(z, \alpha_{s}\right)
$$

where $\sigma_{0}$ is the leading order (LO) partonic cross section, so that the coefficient function is normalized to $\delta(1-z)$ at leading order:

$$
C\left(z, \alpha_{s}\right)=\delta(1-z)+\alpha_{s} C^{(1)}(z)+\alpha_{s}^{2} C^{(2)}(z)+\ldots,
$$

and $z=M^{2} / \hat{s}$ is the variable already mentioned in the introduction. In terms of this coefficient function the cross section eq. (2.1) reads

$$
\sigma\left(\tau, M^{2}\right)=\tau \sigma_{0}\left(M^{2}\right) \int_{\tau}^{1} \frac{d z}{z} \mathscr{L}\left(\frac{\tau}{z}\right) C\left(z, \alpha_{s}\left(M^{2}\right)\right)
$$

which has the form of a Mellin convolution, and factorizes in Mellin space

$$
\sigma\left(N, M^{2}\right)=\int_{0}^{1} d \tau \tau^{N-1} \frac{\sigma\left(\tau, M^{2}\right)}{\tau}=\sigma_{0}\left(M^{2}\right) \mathscr{L}(N) C\left(N, \alpha_{s}\left(M^{2}\right)\right) .
$$

Note that we have used the same symbols, with different arguments, for a function and its Mellin transform; note also that, for convenience, we have indicated with $\sigma\left(N, M^{2}\right)$ the Mellin transform of $\sigma\left(\tau, M^{2}\right) / \tau$.

Soft-gluon resummation is generically performed in $N$-space, where the multiple gluon emission phase-space factorizes. The $N$-space resummed coefficient function (for Higgs and 


\begin{tabular}{|c|c|c|c|c|c|c|}
\hline Notation* & Notation' $^{\prime}$ & $A\left(\alpha_{s}\right)$ & $D\left(\alpha_{s}\right)$ & $\bar{g}_{0}\left(\alpha_{s}\right)$ & $C_{\mathrm{res}} \ni \alpha_{s}^{n} L^{k} \quad \forall n$ & adopted in \\
\hline $\mathrm{LL}$ & LL & 1-loop & - & tree-level & $k=2 n$ & \\
\hline NLL* & NLL & 2-loop & 1-loop & tree-level & $2 n-1 \leq k \leq 2 n$ & \\
\hline NLL & $\mathrm{NLL}^{\prime}$ & 2-loop & 1-loop & 1-loop & $2 n-2 \leq k \leq 2 n$ & \\
\hline NNLL* & NNLL & 3-loop & 2-loop & 1-loop & $2 n-3 \leq k \leq 2 n$ & \\
\hline NNLL & NNLL $^{\prime}$ & 3-loop & 2-loop & 2-loop & $2 n-4 \leq k \leq 2 n$ & Refs. $[1,42]$ \\
\hline $\mathrm{N}^{3} \mathrm{LL}^{*}$ & $\mathrm{~N}^{3} \mathrm{LL}$ & 4-loop & 3-loop & 2-loop & $2 n-5 \leq k \leq 2 n$ & Ref. [43] \\
\hline $\mathrm{N}^{3} \mathrm{LL}$ & $\mathrm{N}^{3} \mathrm{LL}^{\prime}$ & 4-loop & 3-loop & 3-loop & $2 n-6 \leq k \leq 2 n$ & this work \\
\hline
\end{tabular}

Table 1. Orders of logarithmic approximations and accuracy of the predicted $\operatorname{logarithms} L=\log N$. See ref. [44]. Note that the four-loop contribution to $A\left(\alpha_{s}\right)$ is yet unknown and in our study we take a Padé approximation [45].

Drell-Yan production) has the form [39-41]

$$
\begin{aligned}
C_{\text {res }}\left(N, \alpha_{s}\right) & =\bar{g}_{0}\left(\alpha_{s}\right) \exp \overline{\mathcal{S}}\left(\alpha_{s}, N\right), \\
\overline{\mathcal{S}}\left(\alpha_{s}, N\right) & =\int_{0}^{1} d z z^{N-1}\left[\frac{1}{1-z}\left(\int_{\mu_{\mathrm{F}}^{2}}^{M^{2}(1-z)^{2}} \frac{d \mu^{2}}{\mu^{2}} 2 A\left(\alpha_{s}\left(\mu^{2}\right)\right)+D\left(\alpha_{s}\left([1-z]^{2} M^{2}\right)\right)\right)\right]_{+} \\
& =\int_{0}^{1} d z \frac{z^{N-1}-1}{1-z}\left(\int_{\mu_{\mathrm{F}}^{2}}^{M^{2}(1-z)^{2}} \frac{d \mu^{2}}{\mu^{2}} 2 A\left(\alpha_{s}\left(\mu^{2}\right)\right)+D\left(\alpha_{s}\left([1-z]^{2} M^{2}\right)\right)\right), \\
\bar{g}_{0}\left(\alpha_{s}\right) & =1+\sum_{k=1}^{\infty} \bar{g}_{0, k} \alpha_{s}^{k}, \\
A\left(\alpha_{s}\right) & =\sum_{k=1}^{\infty} A_{k} \alpha_{s}^{k}, \quad D\left(\alpha_{s}\right)=\sum_{k=1}^{\infty} D_{k} \alpha_{s}^{k},
\end{aligned}
$$

where $\alpha_{s}=\alpha_{s}\left(\mu_{\mathrm{R}}^{2}\right)$, and $\bar{g}_{0}\left(\alpha_{s}\right)$ does not depend on $N$, but depends implicitly on $\mu_{\mathrm{F}} / M$ and $\mu_{\mathrm{R}} / M$. The function $A\left(\alpha_{s}\right)$ (also called cusp anomalous dimension $\Gamma_{\text {cusp }}$ ) is the numerator of the divergent part of the relevant ${ }^{1}$ diagonal Altarelli-Parisi splitting function,

$$
P\left(z, \alpha_{s}\right)=\frac{A\left(\alpha_{s}\right)}{(1-z)_{+}}+B\left(\alpha_{s}\right) \delta(1-z)+\mathcal{O}\left((1-z)^{0}\right),
$$

and $D\left(\alpha_{s}\right)$ is a process-dependent function. A given logarithmic accuracy is obtained including the functions $A\left(\alpha_{s}\right), D\left(\alpha_{s}\right)$ and $\bar{g}_{0}\left(\alpha_{s}\right)$ up to a given order in eq. (2.7), according to table 1 .

Table 1 shows two notations for the counting of logarithms, usually adopted in different contexts. In particular, in the two notation what is called $\mathrm{N}^{k} \mathrm{LL}$ for $k>0$ represents two different accuracies, and therefore can lead to some confusion. In what we call Notation', the $\mathrm{N}^{k} \mathrm{LL}$ accuracy without decoration corresponds to a logarithmic counting on $\log C_{\text {res }}$,

\footnotetext{
${ }^{1}$ Depending on the considered process, the relevant splitting function can be $P_{g g}$ (Higgs) or $P_{q q}$ (DrellYan); the corresponding $A_{g}\left(\alpha_{s}\right)$ and $A_{q}\left(\alpha_{s}\right)$ functions are simply related by a color factor: $C_{F} A_{g}\left(\alpha_{s}\right)=$ $C_{A} A_{q}\left(\alpha_{s}\right)$. The same color-charge relation holds for $D\left(\alpha_{s}\right): C_{F} D_{\text {Higgs }}\left(\alpha_{s}\right)=C_{A} D_{\text {Drell-Yan }}\left(\alpha_{s}\right)$.
} 
where the inclusion of an additional order in $\bar{g}_{0}\left(\alpha_{s}\right)$, corresponding to $\mathrm{N}^{k} \mathrm{LL}$, does not increase the formal accuracy. However, as shown explicitly in the table, $\mathrm{N}^{k} L L^{\prime}$ predicts an additional term to all orders in the tower of logarithms contained in $C_{\text {res }}$. In practice, when the process is not very close to the physical threshold (i.e., $\tau$ is not close to 1 ), as in all relevant cases, the additional logarithm included at $\mathrm{N}^{k} \mathrm{LL}^{\prime}$ improves the actual accuracy of the result. Therefore, in a wide literature, the $\mathrm{N}^{k} L L^{\prime}$ is simply called $\mathrm{N}^{k} \mathrm{LL}$, as shown in what we call Notation*, where the lower accuracy is denoted with a ${ }^{*}$, although this notation is not widespread. In what follows, we will refer to a logarithmic accuracy according to Notation*.

The three-loop coefficients of $A\left(\alpha_{s}\right)$ and $D\left(\alpha_{s}\right)$ have been known for while (see for instance refs. [45-47]), while the $\mathcal{O}\left(\alpha_{s}^{3}\right)$ contribution to $\bar{g}_{0}\left(\alpha_{s}\right)$ has been recently computed in the infinite top-mass limit [35]. The function $A\left(\alpha_{s}\right)$, however, is needed at four loops in order to achieve full $\mathrm{N}^{3} \mathrm{LL}$ accuracy. This contribution is yet unknown; however, a Padé estimate [45] can suggest the size of its value, and a numerical analysis shows that its impact in a resummed result is essentially negligible. Furthermore, we note that, even without this contribution, the expansion of the $\mathrm{N}^{3} \mathrm{LL}^{\prime}$ resummation to third order in the strong coupling completely reproduces all the soft and constant contributions up to $\mathrm{N}^{3} \mathrm{LO}$, i.e. the four loop coefficient of $A\left(\alpha_{s}\right)$ only enters at order $\alpha_{s}^{4}$.

\section{$2.2 \quad$ Large $N$ limit $-N$-soft}

The Mellin transform in eq. (2.8) is ill defined, because $z$ ranges from 0 to 1 , forcing the argument of $\alpha_{s}$ to be arbitrarily small, therefore crossing the Landau pole. However, the integral can be made convergent by using the explicit solution for the running coupling

$$
\alpha_{s}\left(\mu^{2}\right)=\frac{\alpha_{s}\left(\mu_{\mathrm{R}}^{2}\right)}{X}-\frac{\alpha_{s}^{2}\left(\mu_{\mathrm{R}}^{2}\right)}{X^{2}} \frac{\beta_{1}}{\beta_{0}} \log X+\ldots, \quad X=1+\beta_{0} \alpha_{s}\left(\mu_{\mathrm{R}}^{2}\right) \log \frac{\mu^{2}}{\mu_{\mathrm{R}}^{2}}
$$

to any finite order. In this way, $\overline{\mathcal{S}}$ can be formally written as

$$
\begin{aligned}
\overline{\mathcal{S}}\left(\alpha_{s}, N\right) & =\int_{0}^{1} d z z^{N-1} \overline{\mathcal{S}}\left(\alpha_{s}, z\right)=\sum_{n=1}^{\infty} \alpha_{s}^{n} \sum_{k=0}^{n} b_{n, k} \mathcal{D}_{k}(N) \\
\overline{\mathcal{S}}\left(\alpha_{s}, z\right) & =\sum_{n=1}^{\infty} \alpha_{s}^{n} \sum_{k=0}^{n} b_{n, k} \mathcal{D}_{k}(z)
\end{aligned}
$$

where

$$
\mathcal{D}_{k}(z)=\left(\frac{\log ^{k}(1-z)}{1-z}\right)_{+}
$$

are the usual plus-distributions and

$$
\mathcal{D}_{k}(N)=\int_{0}^{1} d z z^{N-1} \mathcal{D}_{k}(z)=\frac{1}{k+1} \sum_{j=0}^{k+1}\left(\begin{array}{c}
k+1 \\
j
\end{array}\right) \Gamma^{(j)}(1)\left[\frac{d^{k+1-j}}{d \xi^{k+1-j}} \frac{\Gamma(N)}{\Gamma(N+\xi)}\right]_{\xi=0}
$$

their Mellin transform. 
The Mellin transform in eq. (2.13) is usually computed, to any finite logarithmic accuracy, in the large- $N$ limit, leading to an expression of the form

$$
\begin{aligned}
C_{N \text {-soft }}\left(N, \alpha_{s}\right) & =g_{0}\left(\alpha_{s}\right) \exp \mathcal{S}\left(\alpha_{s}, \log N\right), \\
\mathcal{S}\left(\alpha_{s}, \log N\right) & =\left[\frac{1}{\alpha_{s}} g_{1}\left(\alpha_{s} \log N\right)+g_{2}\left(\alpha_{s} \log N\right)+\alpha_{s} g_{3}\left(\alpha_{s} \log N\right)+\alpha_{s}^{2} g_{4}\left(\alpha_{s} \log N\right)+\ldots\right] \\
g_{0}\left(\alpha_{s}\right) & =1+\sum_{k=1}^{\infty} g_{0, k} \alpha_{s}^{k}, \\
g_{i}(\lambda) & =\sum_{k=1}^{\infty} g_{i, k} \lambda^{k}, \quad g_{1,1}=0,
\end{aligned}
$$

where we have introduced a new notation $(N$-soft) for the resummed coefficient function to stress the fact that the large- $N$ limit has been taken. Note that

$$
C_{N \text {-soft }}\left(N, \alpha_{s}\right)=C_{\text {res }}\left(N, \alpha_{s}\right)\left[1+\mathcal{O}\left(\frac{1}{N}\right)\right]
$$

The functions $g_{i}, i=1,2,3,4$ can be found explicitly for many processes in ref. [45]. A given $\mathrm{N}^{k} \mathrm{LL}$ accuracy is obtained from eq. (2.17) including $g_{i}$ up to $i=k+1$, and $g_{0}\left(\alpha_{s}\right)$ up to the same order as $\bar{g}_{0}\left(\alpha_{s}\right)$, see table 1 . The function $\mathcal{S}\left(\alpha_{s}, \log N\right)$ can be written as

$$
\mathcal{S}\left(\alpha_{s}, \log N\right)=\sum_{n=1}^{\infty} \alpha_{s}^{n} \sum_{k=0}^{n} b_{n, k} \mathcal{D}_{k}^{\log }(N),
$$

where the coefficients $b_{n, k}$ are the same as in eq. (2.13), and the functions $\mathcal{D}_{k}^{\log }(N)$ are the large- $N$ limit of $\mathcal{D}_{k}(N)$ expressed in terms of $\log N$, and neglecting constant terms and terms suppressed by powers of $1 / N$ :

$$
\mathcal{D}_{k}^{\log }(N)=\frac{1}{k+1} \sum_{j=0}^{k}\left(\begin{array}{c}
k+1 \\
j
\end{array}\right) \Gamma^{(j)}(1) \log ^{k+1-j} \frac{1}{N} .
$$

Here $\Gamma^{(j)}(x)$ is the $j$-th derivative of the Euler Gamma function. For completeness, we also report the functional form of the momentum space conjugates of the $\mathcal{D}_{k}^{\log }(N)$ functions:

$$
\mathcal{D}_{k}^{\log }(z)=\left(\frac{\log ^{k} \log \frac{1}{z}}{\log \frac{1}{z}}\right)_{+}, \quad \text { with } \quad \mathcal{D}_{k}^{\log }(N)=\int_{0}^{1} d z z^{N-1} \mathcal{D}_{k}^{\log }(z) .
$$

The relation between the function $g_{0}$ and the constant that multiplies the resummed exponent in eq. (2.7), namely $\bar{g}_{0}$, is

$$
g_{0}\left(\alpha_{s}\right)=\bar{g}_{0}\left(\alpha_{s}\right) \exp \left[\sum_{n=1}^{\infty} \alpha_{s}^{n} \sum_{k=0}^{n} b_{n, k} d_{k}\right]
$$

with

$$
d_{k}=\lim _{N \rightarrow \infty}\left[\mathcal{D}_{k}(N)-\mathcal{D}_{k}^{\log }(N)\right]=\frac{\Gamma^{(k+1)}(1)}{k+1},
$$

(for further details, see ref. [37]). 


\subsection{Prescriptions for the Landau pole}

The resummed coefficient function $C_{N \text {-soft }}$, eq. (2.17), although well defined in $N$ space, cannot be directly used for computing the corresponding hadron-level cross section because its inverse Mellin transform does not exist. Indeed, the functions $g_{i}\left(\alpha_{s} \log N\right)$ have a branch-cut for real $N>N_{L}=\exp \frac{1}{2 \beta_{0} \alpha_{s}}$ originating from the Landau pole of the running coupling, while Mellin transformation has a convergence abscissa [48]. On the other hand, if $C_{N \text {-soft }}$ is expanded in powers of $\alpha_{s}$, the inverse Mellin transform exists to any finite order, but the resulting series is divergent [49]. Therefore, a prescription is needed to compute physical observables from eq. (2.17).

The most used prescription is the so called Minimal Prescription (MP), proposed long ago [48]. It consists on a simple modification of the Mellin inversion integral, and has the advantage of having a fast numerical implementation. More details are given in appendix A.1. More recently, another prescription based on a Borel summation of the

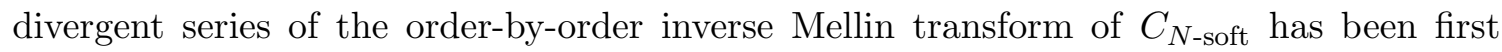
proposed in ref. [49], and refined and extended in refs. [50-53]. This prescription, called Borel Prescription (BP), is typically slower but more flexible. More details are given in appendix A.2.

It turns out that the numerical difference between the two prescriptions is small, being totally negligible for Higgs phenomenology [53]. The reason is that, for the kinematic configurations typical of high-energy colliders, i.e. $\tau \ll 1$, the series is behaving in a perturbative way. Hence, the all-order nature of the series does not play any role, and, a fortiori, the way the divergence of the series is dealt with is immaterial. We believe that expanding $C_{N \text {-soft }}$ to a sufficiently large, but finite, order in $\alpha_{s}$ and inverting exactly would lead to a result virtually identical to the all-order $\mathrm{MP}$ or $\mathrm{BP}$ results.

One reason why the effective equivalence of $\mathrm{MP}$ and $\mathrm{BP}$ does not appear clearly in the literature is the fact that, within the BP, the form of the soft terms that are resummed can be easily modified, and this has been always done for phenomenological application, thereby giving a result which differs from the MP one. A discussion on the form of the soft terms will be performed in section 3. Here, we just want to mention that, for practical applications, the choice of the prescription will be mainly dictated by its flexibility and numerical efficiency.

Details on the practical implementation of the prescriptions, as well as details on a new version of the BP acting directly on the Sudakov exponent $\mathcal{S}\left(\alpha_{s}, \log N\right)$, are given in appendix A.

\section{Soft terms and analyticity conditions}

Soft gluon resummation fixes the coefficients $b_{n, k}$ of the soft terms in eq. (2.22), however it does not fix the functional form of these contributions. In particular, choices that at large $N$ only differ by terms suppressed by factors of $1 / N$ are equally acceptable.

It has been pointed out $[37,54]$ that the actual form of the soft terms is very important, and different choices would lead to very different accuracies, in particular when the considered process is far from the physical threshold. 
In our previous analysis [37], we studied the analyticity properties of coefficient functions in $N$ space and we arrived at an optimal choice of the soft terms. We used this improved large- $N$ behavior, together with the knowledge of the rightmost singularity at finite $N$ from high-energy resummation to compute an approximate expression for the $\mathrm{N}^{3} \mathrm{LO}$ Higgs production cross section [37, 38].

In this section, we briefly review the two main theoretical ingredients that go into the construction of our resummation formula, namely a choice of soft terms that respects the singularity structure of coefficient functions and the improvement related to the inclusion of collinear contributions.

\subsection{Functional form of the soft terms}

The $N$-soft resummed exponent eq. (2.22) is an infinite sum of contributions each of which has a logarithmic branch cut starting at $N=0$, which is not compatible with the known singularity structure of coefficient functions. However, this problem is an artefact of the large $N$ approximation which we have employed in going from $\overline{\mathcal{S}}$, eq. (2.13), to $\mathcal{S}$, eq. (2.22). Indeed, the resummed exponent $\overline{\mathcal{S}}$ is written as an infinite sum of $\mathcal{D}_{k}(N)$ functions, whose singularity structure is compatible with the one of fixed-order calculations.

Furthermore, as we discussed at length in ref. [37], we can improve on the use of $\mathcal{D}_{k}(N)$ by noticing that the correct kinematic limit of the $\mu^{2}$ integration in eq. (2.8) is actually $M^{2} \frac{(1-z)^{2}}{z}$. This consideration leads to the following choice for the soft terms in momentum space

$$
\hat{\mathcal{D}}_{k}(z)=\mathcal{D}_{k}(z)+\frac{\log ^{k} \frac{1-z}{\sqrt{z}}}{1-z}-\frac{\log ^{k}(1-z)}{1-z}=\left[\frac{d^{k}}{d \xi^{k}}\left(z^{-\xi / 2}\left[(1-z)^{\xi-1}\right]_{+}\right)\right]_{\xi=0},
$$

from which we can easily compute the functions $\hat{\mathcal{D}}_{k}(N)$, which enter our resummation formula:

$$
\hat{\mathcal{D}}_{k}(N)=\int_{0}^{1} d z z^{N-1} \hat{\mathcal{D}}_{k}(z)=\frac{1}{k+1} \sum_{j=0}^{k+1}\left(\begin{array}{c}
k+1 \\
j
\end{array}\right) \Gamma^{(j)}(1)\left[\frac{d^{k+1-j}}{d \xi^{k+1-j}} \frac{\Gamma(N-\xi / 2)}{\Gamma(N+\xi / 2)}\right]_{\xi=0} .
$$

Note that in eq. (3.1) we have chosen to apply the plus prescription only to the first term, singular in $z=1$, which is the natural choice in fixed order calculations. In this way, $\hat{\mathcal{D}}_{k}(N)$ differs from $\mathcal{D}_{k}(N)$ only by terms vanishing at large $N$ :

$$
\lim _{N \rightarrow \infty}\left[\hat{\mathcal{D}}_{k}(N)-\mathcal{D}_{k}(N)\right]=0 .
$$

Adopting this form for the soft terms in all the terms generated in eq. (2.8) (hence also those generated by the $D\left(\alpha_{s}\right)$ term), we arrive at the expression

$$
\overline{\mathcal{S}}\left(\alpha_{s}, N\right) \rightarrow \sum_{n=1}^{\infty} \alpha_{s}^{n} \sum_{k=0}^{n} b_{n, k} \hat{\mathcal{D}}_{k}(N)
$$

which represents the first improved version of eq. (2.13). This is not yet our final formula, as we are going to discuss. 


\subsection{Altarelli-Parisi contributions}

We have already discussed the origin of the coefficient $A\left(\alpha_{s}\right)$ in the resummation formula eq. (2.8): it is the coefficient of the soft-enhanced part of the relevant Altarelli-Parisi splitting function eq. (2.11), where $P=P_{g g}$ in the case of Higgs production. In resummed calculation, the coefficient $B\left(\alpha_{s}\right)$ in eq. (2.11) is also retained at the appropriate accuracy because it corresponds to a constant term in $N$-space, while contributions that vanish as $z \rightarrow 1$ are usually neglected in eq. (2.8). However, an important class of these subleading corrections can be taken into account to all orders, essentially because the full leading order anomalous dimension exponentiates $[54,55]$.

However, the $1 / z$ pole present in the $\mathrm{LO}$ gluon-gluon splitting function would introduce spurious singularities in the resummed coefficient function at $N=1$ [37]. Nevertheless, the expansion of $(1-z) P_{g g}^{(0)}(z)$ (with $P_{g g}^{(0)}(z)$ being the LO gluon-gluon Altarelli-Parisi splitting function) in powers of $1-z$ to any finite order is not singular in $z=0$, and therefore does not affect the singularity structure around $N=1$. The expansion up to second order reads

$$
\begin{aligned}
(1-z) P_{g g}^{(0)}(z) & =A_{1}\left[1-(1-z)+2(1-z)^{2}+\mathcal{O}\left((1-z)^{3}\right)\right] \\
& =A_{1}\left[2-3 z+2 z^{2}\right]+\mathcal{O}\left((1-z)^{3}\right),
\end{aligned}
$$

where $A_{1}=C_{A} / \pi$ is the first coefficient in the expansion of $A\left(\alpha_{s}\right)$, eq. (2.10). Note that the third order term in the expansion is accidentally zero. Upon Mellin transformation, multiplication by the factor $2-3 z+2 z^{2}$ results into the shift

$$
\left[2-3 z+2 z^{2}\right] f(z) \stackrel{\text { Mellin }}{\longrightarrow} \mathcal{A P}_{2}[f(N)] \equiv 2 f(N)-3 f(N+1)+2 f(N+2),
$$

where we have introduce the Altarelli-Parisi $\left(\mathcal{A P}_{2}\right)$ operator to second order for future convenience.

The $A_{1}$ term in eq. (2.8) is responsible for the tower of LL terms, namely terms $\alpha_{s}^{n} \mathcal{D}_{2 n-1}(z)$ to all orders $n$ in $C\left(z, \alpha_{s}\right)$. When $A_{1}$ is replaced with the expansion eq. (3.5), logarithmic terms suppressed by powers of $(1-z)$ are generated to all orders. The towers of LL suppressed logarithms, namely terms of the form $(1-z)^{k-1} \alpha_{s}^{n} \log ^{2 n-1}(1-z)$ in $C\left(z, \alpha_{s}\right)$, with $k$ running from one up to the order of the expansion of $(1-z) P_{g g}^{(0)}(z)$, are correctly predicted to all orders [56]. However, the simple inclusion of additional information from Altarelli-Parisi splitting functions is not enough to predict terms beyond these LL towers.

Nevertheless, we have shown in ref. [37] that including the expansion of $(1-z) P_{g g}^{(0)}(z)$ up to second order for all the terms in the Sudakov exponent eq. (2.14) leads to an approximation of the exact fixed-order terms which is very good in a wide range of $N$ values, down to values where high-energy terms (not considered in this work) start being relevant. This is achieved when the soft terms introduced in section 3.1 are used. Therefore, the inclusion of such second order expansion for all the terms in eq. (2.8) amounts to replacing in eq. (3.4)

$$
\hat{\mathcal{D}}_{k}(N) \rightarrow \mathcal{A} \mathcal{P}_{2}\left[\hat{\mathcal{D}}_{k}(N)\right]=2 \hat{\mathcal{D}}_{k}(N)-3 \hat{\mathcal{D}}_{k}(N+1)+2 \hat{\mathcal{D}}_{k}(N+2) .
$$

Note that the inclusion of terms of order $(1-z)^{4}$ and higher in the expansion of $(1-z) P_{g g}^{(0)}(z)$ does not affect the results significantly [37]. At resummed level, the operator $\mathcal{A P}_{2}$ in eq. (3.7) can be applied directly to the Sudakov exponent. 


\section{$3.3 \quad A$-soft 2}

We are now ready to present our resummed expression. We name it ${ }^{2} A$-soft 2 , because it takes into account the analyticity properties discussed in section 3.1 and it includes the first two terms of the $(1-z)$ expansion of the LO splitting function, as detailed in section 3.2:

$$
C_{A \text {-soft }}\left(N, \alpha_{s}\right)=\bar{g}_{0}\left(\alpha_{s}\right) \exp \sum_{n=1}^{\infty} \alpha_{s}^{n} \sum_{k=0}^{n} b_{n, k} \mathcal{A} \mathcal{P}_{2}\left[\hat{\mathcal{D}}_{k}(N)\right]
$$

Note that because the difference between the functions $\hat{\mathcal{D}}_{k}(N)$ and $\mathcal{D}(N)$ vanishes at large $N$, eq. (3.3), the constant term $\bar{g}_{0}$ in eq. (3.8) coincides with the one in eq. (2.7). As in the case of $N$-soft resummation previously discussed, the series that defines $C_{A \text {-soft }}$ in eq. (3.8) is divergent. This series is summed using the Borel Prescription ${ }^{3}$ detailed in appendix A.2, which leads to the following result

$$
\begin{aligned}
C_{A-\text { soft }_{2}}\left(N, \alpha_{s}\right)=\bar{g}_{0}\left(\alpha_{s}\right) \exp & \left\{\frac{1}{2 \pi i} \int_{0}^{\frac{W}{2 \beta_{0} \alpha_{s}}} d w e^{-w} \oint \frac{d \xi}{\xi} \mathcal{S}\left(\alpha_{s},-\frac{w}{\xi}\right)\right. \\
\times & {\left.\left[\mathcal{A} \mathcal{P}_{2}\left[\frac{\Gamma(N-\xi / 2)}{\Gamma(N+\xi / 2)}\right]-\frac{1}{\Gamma(1+\xi)}\right]\right\}, }
\end{aligned}
$$

where $W$ is a cut-off, minimally set to $W=2$. The inverse Mellin transform of the above result is finite, and can then be used to compute hadron-level cross sections.

We have noticed in previous papers [37, 38] that one of interesting consequences of using $A$-soft 2 instead of $N$-soft is a better perturbative behavior, which is partially due to the resulting constant multiplying the resummation, $\bar{g}_{0}$ or $g_{0}$ respectively. In fact, while the perturbative expansion of the function $g_{0}$ is known to be poor, driving large fixed-order corrections to the Higgs production, the function $\bar{g}_{0}$ has a much more stable perturbative expansion [37, 38]. The relation between the two constants is given in eq. (2.25), from which it is clear that by using $\bar{g}_{0}$, we are effectively exponentiating and, hence, resumming, part of the constant contribution. This is similar in spirit to the so-called $\pi^{2}$-resummation discussed in refs. [57-63] for Higgs and Drell-Yan processes.

We can also go a step further and try to exponentiate the whole constant term [63]. To $\mathrm{N}^{3} \mathrm{LL}$ accuracy this amounts to replacing in the resummed expressions $\bar{g}_{0}\left(\alpha_{s}\right)$ with

$$
\bar{G}_{0}\left(\alpha_{s}\right)=\exp \left[\alpha_{s} \bar{g}_{0,1}+\alpha_{s}^{2}\left(\bar{g}_{0,2}-\frac{\bar{g}_{0,1}^{2}}{2}\right)+\alpha_{s}^{3}\left(\bar{g}_{0,3}-\bar{g}_{0,1} \bar{g}_{0,2}+\frac{\bar{g}_{0,1}^{3}}{3}\right)+\mathcal{O}\left(\alpha_{s}^{4}\right)\right] \text {. }
$$

By construction the difference between $\bar{g}_{0}$ and $\bar{G}_{0}$ is $\mathcal{O}\left(\alpha_{s}^{4}\right)$ and hence beyond N ${ }^{3} \mathrm{LL}$ accuracy considered here. Because of the good convergence of the perturbative expansion of $\bar{g}_{0}$ we do not expect the result obtained with $\bar{G}_{0}$ to be much different compared to the one obtained with $\bar{g}_{0}$. We will further comment on this in section 4 , where we present our hadron-level results.

\footnotetext{
${ }^{2}$ This choice was called simply soft 2 in ref. [37], where an average of soft $_{1}$ and soft $_{2}$ was used.

${ }^{3}$ The MP cannot be used in this case, because it is not flexible enough to reproduce the desired soft terms.
} 


\section{$3.4 \psi$-soft 2}

Before turning our attention to phenomenology, we discuss an alternative option for the soft terms, which is very similar to the one used in our improved result $A$-soft . $_{2}$. We first write $\hat{\mathcal{D}}_{k}(N)$ in eq. (3.2) as [53]

$$
\begin{aligned}
\hat{\mathcal{D}}_{k}(N) & =\frac{1}{k+1} \sum_{j=0}^{k+1}\left(\begin{array}{c}
k+1 \\
j
\end{array}\right) \Gamma^{(j)}(1)\left[-\psi_{0}(N)\right]^{k} \times\left[1+\mathcal{O}\left(\frac{1}{N^{2}}\right)\right] \\
& =\frac{\Gamma^{(k+1)}(1)}{k+1}+\mathcal{D}_{k}^{\log }\left(\exp \psi_{0}(N)\right) \times\left[1+\mathcal{O}\left(\frac{1}{N^{2}}\right)\right],
\end{aligned}
$$

where $\psi_{0}(N)=\Gamma^{\prime}(N) / \Gamma(N)$ is the DiGamma function. Thus, except for the constant term, the functions $\hat{\mathcal{D}}_{k}(N)$ are equivalent to the corresponding $\mathcal{D}_{k}^{\log }(N)$ after the replacement $\log N \rightarrow \psi_{0}(N)$, up to corrections of order $1 / N^{2}$. This means that eq. (2.17) can be upgraded by simply replacing $\log N$ with $\psi_{0}(N)$, thereby restoring the analyticity properties of the coefficient function.

Therefore, we propose a new prescription, called $\psi$-soft 2 , where we also include AltarelliParisi improvement,

$$
C_{\psi \text {-soft } 2}\left(N, \alpha_{s}\right)=g_{0}\left(\alpha_{s}\right) \exp \left\{\mathcal{A} \mathcal{P}_{2}\left[\mathcal{S}\left(\alpha_{s}, \psi_{0}(N)\right)\right]\right\},
$$

which has the advantage of having almost all the good properties captured by $A$-soft 2 , while being numerically very fast, because it can be computed using the MP. In fact, any existing soft-gluon resummation code can be easily upgraded to $\psi$ - $\mathrm{soft}_{2}$.

However, eq. (3.12) has a clear disadvantage with respect to $A$-soft 2 , eq. (3.9), namely the presence of the function $g_{0}$ rather than $\bar{g}_{0}$. We have already commented in section 3.3 about the poor perturbative behavior of $g_{0}$ compared to that of $\bar{g}_{0}$. Here we limit ourselves to mention that, in this case, writing $g_{0}$ in exponential form

$$
G_{0}\left(\alpha_{s}\right)=\exp \left[\alpha_{s} g_{0,1}+\alpha_{s}^{2}\left(g_{0,2}-\frac{g_{0,1}^{2}}{2}\right)+\alpha_{s}^{3}\left(g_{0,3}-g_{0,1} g_{0,2}+\frac{g_{0,1}^{3}}{3}\right)+\mathcal{O}\left(\alpha_{s}^{4}\right)\right],
$$

as done for $\bar{g}_{0}$ in eq. (3.10), can improve significantly the perturbative stability of the resummed result. We anticipate that a phenomenological study shows that, after exponentiation of both $g_{0}$ and $\bar{g}_{0}$, results obtained with $A$-soft ${ }_{2}$ and $\psi$-soft 2 are very similar.

\section{Hadron-level results}

In this section we present numerical results for the Higgs production cross section in the gluon fusion channel at the LHC. Our result correctly accounts for finite top mass $\left(m_{\mathrm{t}}=\right.$ $172.5 \mathrm{GeV}$ ) at NNLO [10-15] and NNLL. We also include bottom and charm masses in the LO prefactor (thus changing the overall normalization) and in the NLO contributions, using the results of ref. [64]. The dependence on bottom and charm masses in the resummation is NLL accurate. For consistency with the NNLO set of parton distribution functions NNPDF2.3 [65] with $\alpha_{s}\left(m_{\mathrm{Z}}\right)=0.118$, that we adopt for our phenomenological analysis, we use $m_{\mathrm{b}}=4.75 \mathrm{GeV}$ and $m_{\mathrm{c}}=1.41 \mathrm{GeV}$. We refer the reader to ref. [66] for a discussion about the role of $\mathrm{N}^{3} \mathrm{LO}$ parton densities. We use $m_{\mathrm{H}}=125 \mathrm{GeV}$. 

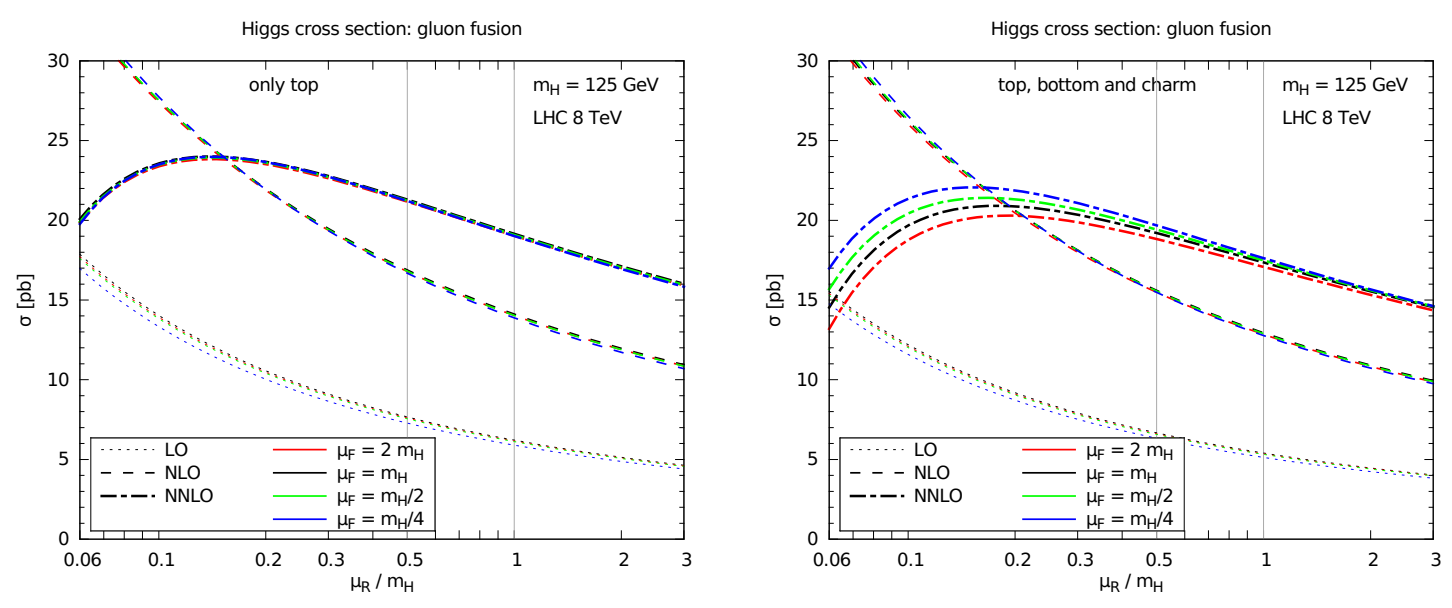

Figure 1. Renormalization scale dependence of LO, NLO and NNLO cross section. We show the effect of including just the top (left panel) and also bottom and charm (right panel) in the loop. The hadronic center of mass energy is $\sqrt{s}=8 \mathrm{TeV}$.

\subsection{A study of the scale dependence}

In order to make contact with our previous work [37], we start by showing in figure 1 the cross section as a function of $\mu_{\mathrm{R}}$ at LO, NLO, and NNLO with only top included in the loop (left panel) and with also bottom and charm (right panel). The results have been computed using the code ggHiggs. The collider energy is $\sqrt{s}=8 \mathrm{TeV}$. We use different colors for different values of $\mu_{\mathrm{F}}$ in all curves, for $\mu_{\mathrm{F}}=\{2,1,1 / 2,1 / 4\} m_{\mathrm{H}}$. We show four choices because the typical scale variation is by a factor of two about its central value, but the central value is sometimes suggested to be $m_{\mathrm{H}}$ (e.g. [1]) and sometimes $m_{\mathrm{H}} / 2$ (e.g. [67]). It is interesting to observe that fixed-order results with only the top quark in the loop, lefthand plot of figure 1 , barely depend on $\mu_{\mathrm{F}}$ : this is due to a compensation between different channels (mainly $g g$ and $q g$ channels) [37]. However, this cancellation is not as perfect at NNLO, when we introduce bottom and charm contributions, right-hand plot of figure 1 . This happens because in our framework, these corrections are correctly implemented only at NLO and there are no $m_{\mathrm{b}}, m_{\mathrm{c}}$ dependent contributions at $\mathcal{O}\left(\alpha_{s}^{2}\right)$ to compensate the NLO $\mu_{\mathrm{F}}$ dependence. We observe that the main effect of including bottom and charm in the loop is to significantly reduce the cross section.

We now move to resummation. In order to study the effect of different logarithmic orders, we show in figure 2 the resummation at LL, NLL, NNLL and $\mathrm{N}^{3} \mathrm{LL}$ accuracy, ${ }^{4}$ always matched to the same NNLO contribution, as a function of $\mu_{\mathrm{R}}$, for fixed $\mu_{\mathrm{F}}=m_{\mathrm{H}}$. We also show, for comparison, LO, NLO and NNLO curves. The fixed order results have been computed using the code ggHiggs, while for the resummation we have written a new code called ResHiggs. The plots show our best prediction, $A$-soft ${ }_{2}$, with $\bar{g}_{0}$ (left panel) and its exponentiated version $\bar{G}_{0}$ (right panel). It is interesting to observe that exponentiating $\bar{g}_{0}$ leads to a flatter resummed result, thereby suggesting that its exponentiation is probably

\footnotetext{
${ }^{4}$ We are adopting Notation*, see table 1 , so $\mathrm{N}^{3} \mathrm{LL}$ is the currently highest possible accuracy.
} 

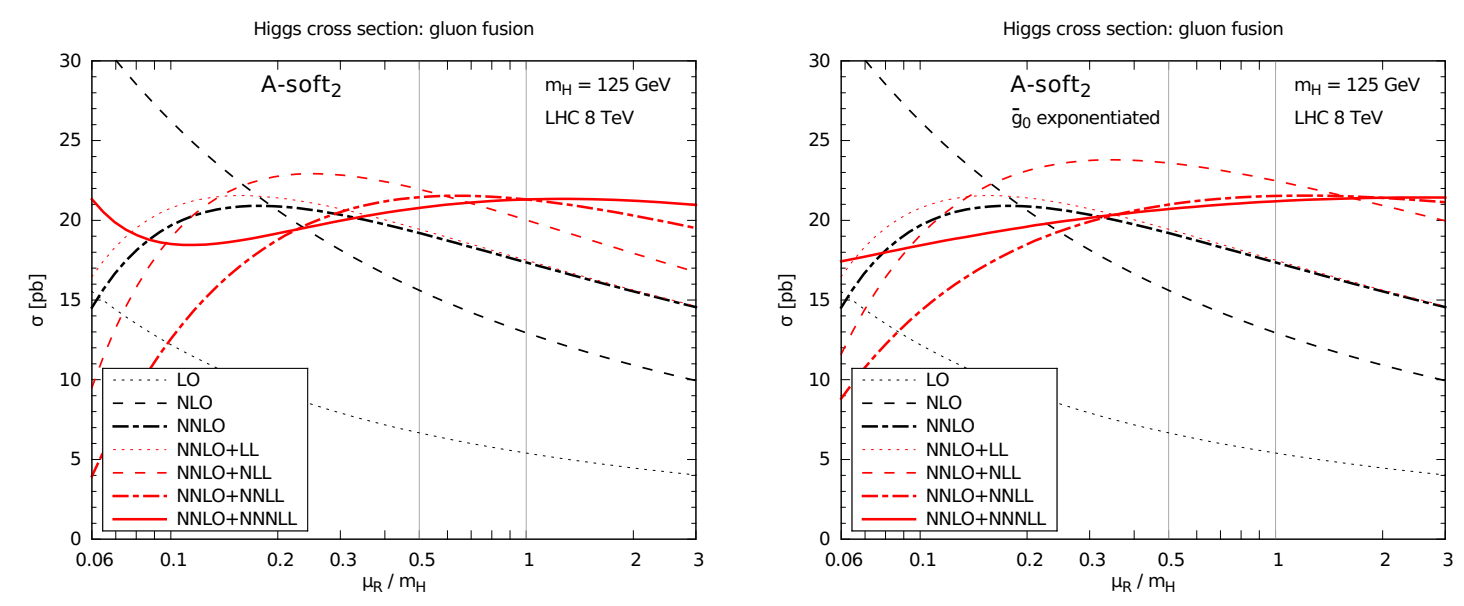

Figure 2. Our best prescription for the resummation, namely $A$-soft ${ }_{2}$ described in section 3.3, plotted as a function of the renormalization scale $\mu_{\mathrm{R}}$. The factorization scale is $\mu_{\mathrm{F}}=m_{\mathrm{H}}$. We show fixed-order results as well as resummed ones. The plot on the left is obtained with the overall constant $\bar{g}_{0}$, while the one on the right with its exponentiated version $\bar{G}_{0}$, as defined in eq. (3.10).
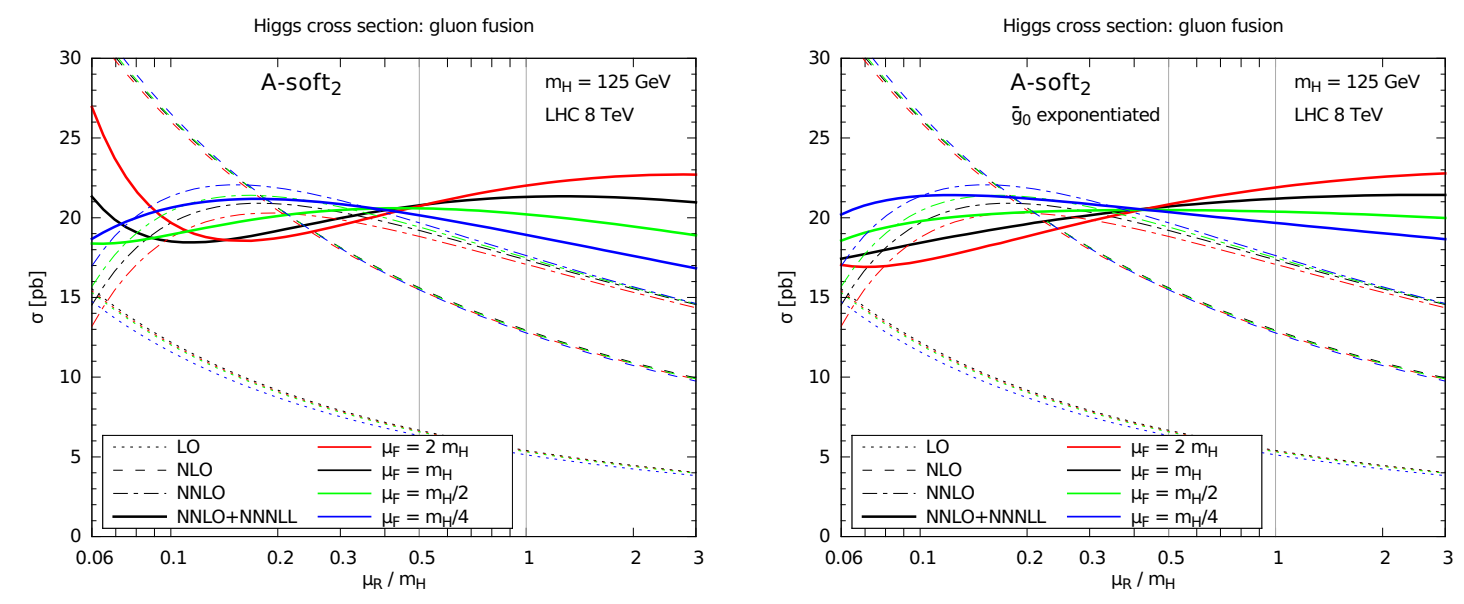

Figure 3. Our best result, namely $\mathrm{NNLO}+\mathrm{N}^{3} \mathrm{LL}$ with the $A$-soft ${ }_{2}$ resummation described in section 3.3, plotted as a function of the renormalization scale $\mu_{\mathrm{R}}$. Different colors correspond to different choices of the factorization scale is $\mu_{\mathrm{F}}$. The plot on the left is obtained with the overall constant $\bar{g}_{0}$, while the one on the right with its exponentiated version $\bar{G}_{0}$, as defined in eq. (3.10).

improving the convergence of the series. We also observe that, in any case, the $\mathrm{N}^{3} \mathrm{LL}$ result is very similar in both cases over a wide range of scales, so the exponentiation of $\bar{g}_{0}$ does not change significantly the final result, as we have anticipated at the end of section 3.3. In both cases, we note that the inclusion of soft-gluon resummation at $\mathrm{N}^{3} \mathrm{LL}$ significantly reduces the $\mu_{\mathrm{R}}$ scale uncertainty of fixed-order results and of previous resummed orders.

In figure 3 we concentrate on NNLO $+\mathrm{N}^{3} \mathrm{LL}$ and also show the effect of varying $\mu_{\mathrm{F}}$. Since the resummation involves only the $g g$ channel, the resummed result depends more 

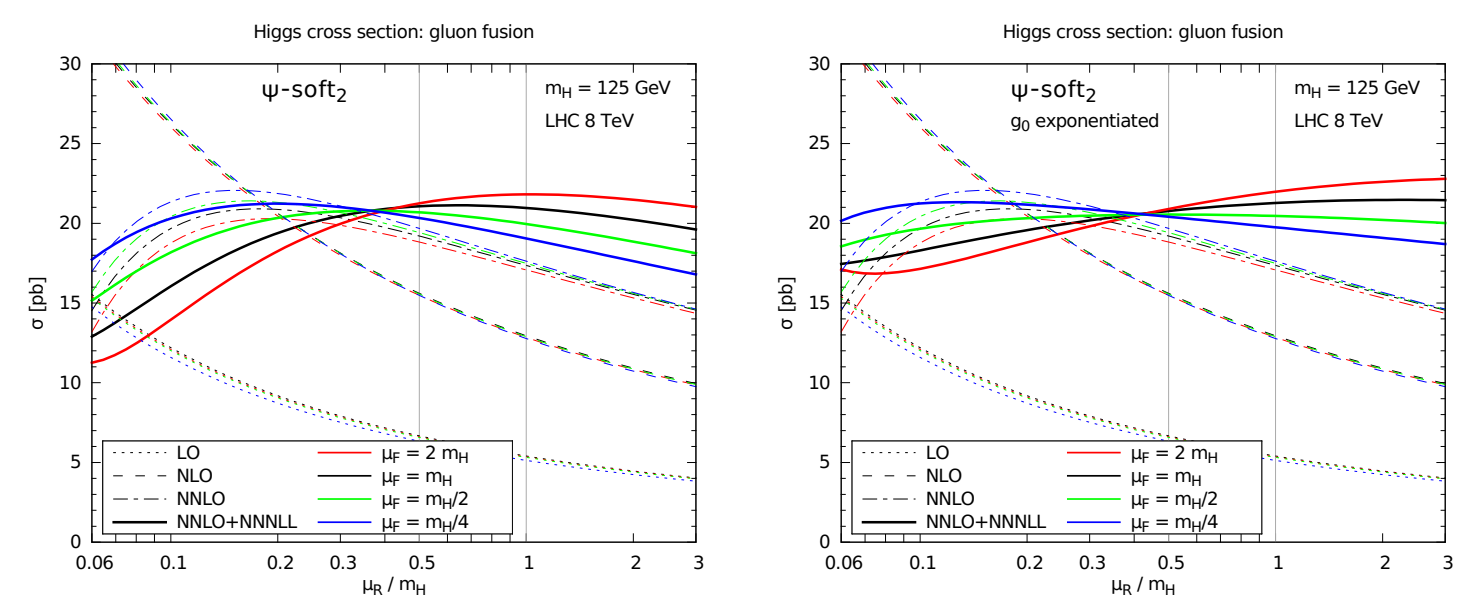

Figure 4. NNLO $+\mathrm{N}^{3} \mathrm{LL}$ for $\psi$-soft ${ }_{2}$ described in section 3.4, plotted as a function of the renormalization scale $\mu_{\mathrm{R}}$. Different colors correspond to different choices of the factorization scale is $\mu_{\mathrm{F}}$. The plot on the left is obtained with the overall constant $g_{0}$, while the one on the right with its exponentiated version $G_{0}$.

significantly on the scale $\mu_{\mathrm{F}}$, although formally such dependence is of order $\alpha_{s}^{3}$ with respect to the Born cross section. Over a range of roughly a factor of 2 about $\mu_{\mathrm{R}}=m_{\mathrm{H}} / 2$ the results with (right panel) or without (left panel) exponentiation of $\bar{g}_{0}$ are very similar, while they differ (and are more sensitive to $\mu_{\mathrm{F}}$ ) for more extreme choices of $\mu_{\mathrm{R}}$ (especially at small $\mu_{\mathrm{R}}$ ). In these regions, the result obtained exponentiating $\bar{g}_{0}$ looks more sensible and stable, suggesting, once again, that exponentiating $\bar{g}_{0}$ provides a more stable result. Moreover, we notice that NNLO $+\mathrm{N}^{3} \mathrm{LL}$ result with $\mu_{\mathrm{F}}=m_{\mathrm{H}} / 2$ barely depends on $\mu_{\mathrm{R}}$. We also observe that resummed curves for different values of $\mu_{\mathrm{F}}$ approximately coincide for a value of $\mu_{\mathrm{R}}$ slightly smaller than $m_{\mathrm{H}} / 2$.

In figure 4 we show the same plots as in figure 3 , but this time obtained with the $\psi$ soft $_{2}$ prescription. Since now the constant function in front of the exponential is $g_{0}$ rather than $\bar{g}_{0}$, we can expect a result different from that of $A$-soft 2 , when $g_{0}$ is not exponentiated (left panel). However, the result with $G_{0}$ (right panel) is very similar to the analogous

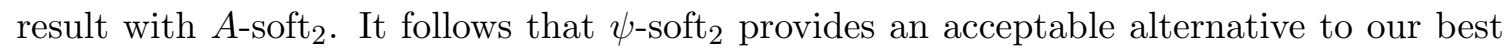

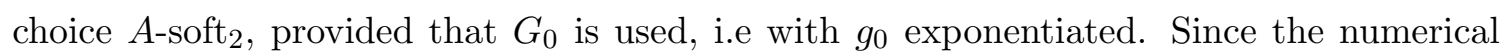
implementation of $\psi$-soft 2 is much faster than that of $A$-soft 2 , its usage can be convenient.

We show the result of the more traditional $N$-soft resummation in figure 5 . This is interesting because the value of the Higgs production cross section which is currently recommended by the Higgs Cross section Working Group [68, 69] is based on refs. [42], which includes $N$-soft resummation to NNLL accuracy. In this case the difference between $\mathrm{N}^{3} \mathrm{LL}$ and NNLL is bigger than it was for $A$-soft 2 , as it is shown on the left-hand plot of figure 5. This difference is partly due to the fact that $g_{0,3}$ is much larger than $\bar{g}_{0,3}[37,38]$. The dependence of renormalization and factorization scale at $\mathrm{N}^{3} \mathrm{LL}$ is comparable $\psi$-soft 2 when $g_{0}$ is not exponentiated; however, the typical increase of the cross section is smaller than what we find with our result. 

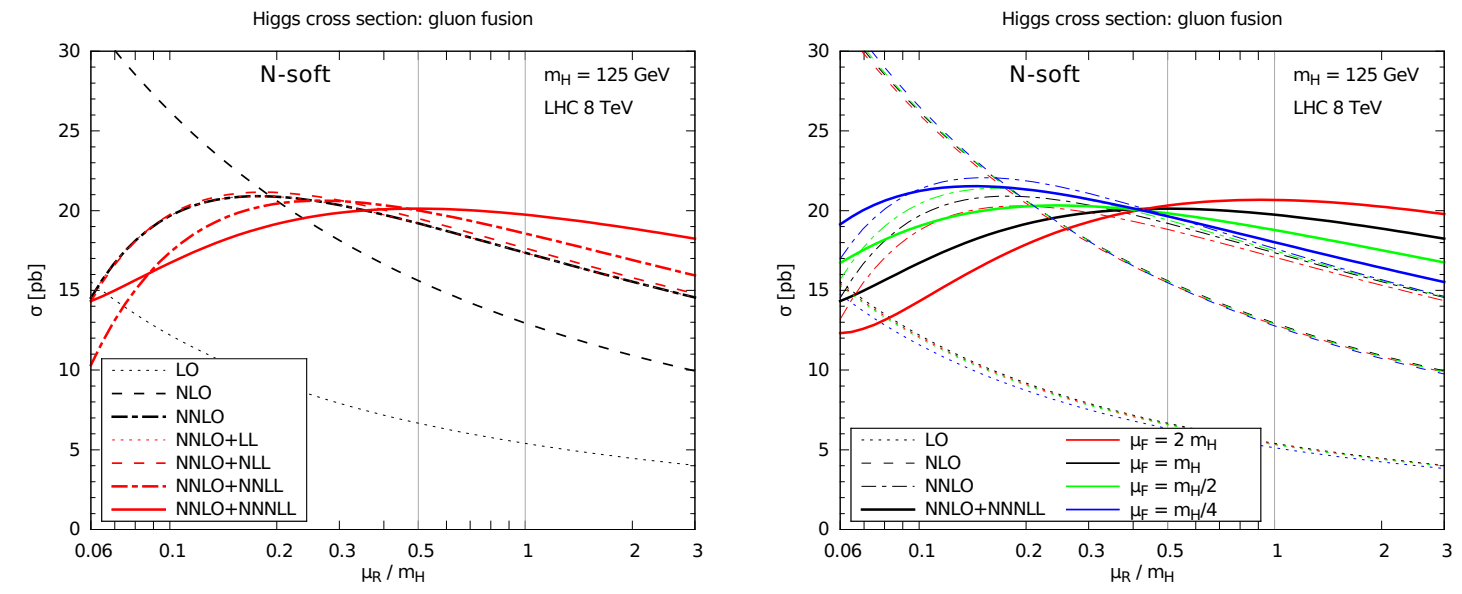

Figure 5. Result for $N$-soft resummation. On the left we show the resummation at different accuracies, always matched to the same NNLO result, for $\mu_{\mathrm{F}}=m_{\mathrm{H}}$, as a function of $\mu_{\mathrm{R}}$. On the right we focus on the $\mathrm{NNLO}+\mathrm{N}^{3} \mathrm{LL}$ result and we also vary $\mu_{\mathrm{F}}$.

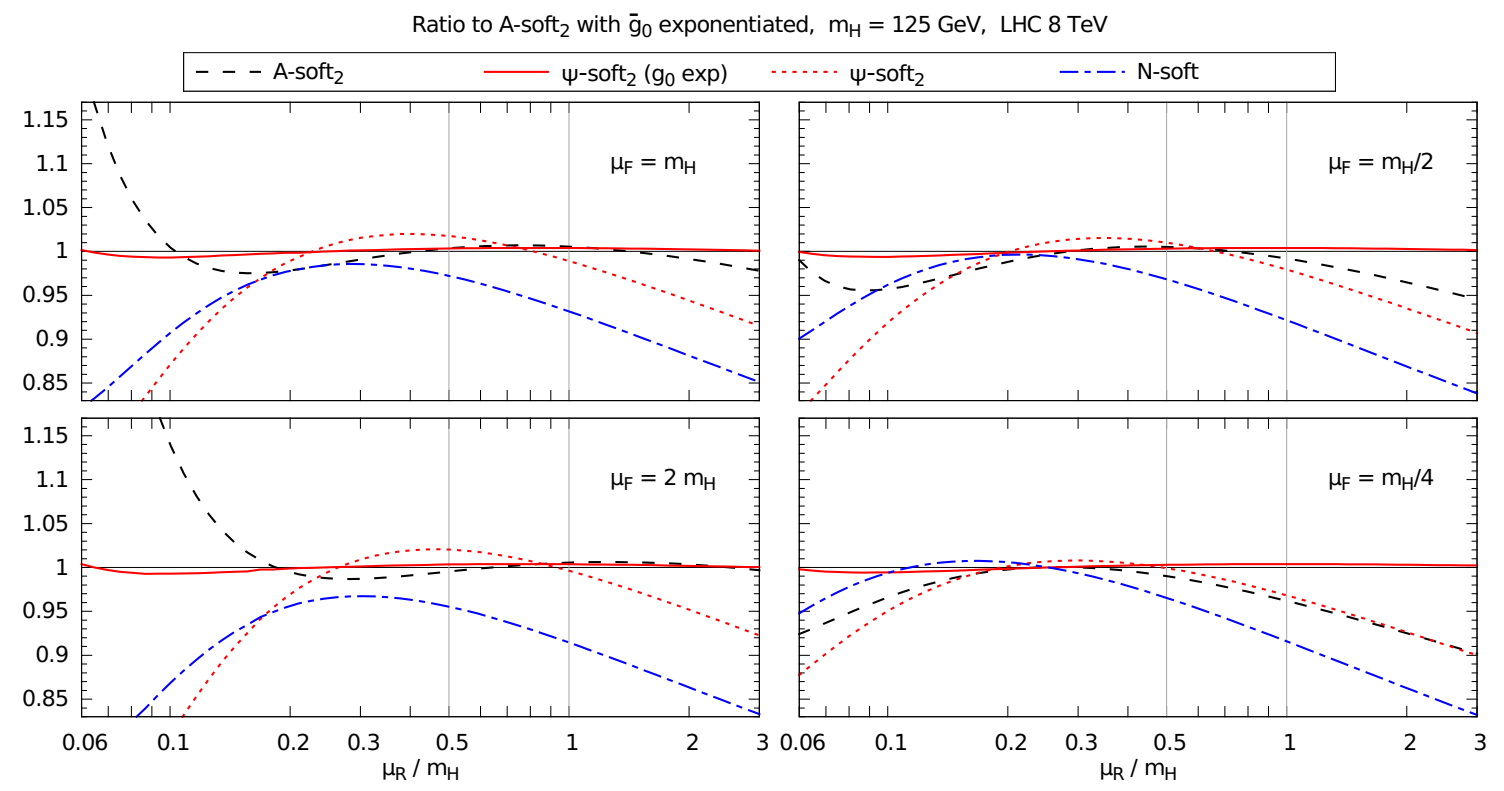

Figure 6. Ratios of different resummed results to our best prediction $A$-soft ${ }_{2}$ with the exponentiated constant $\bar{G}_{0}$, plotted as a function of $\mu_{\mathrm{R}}$, for different choices of $\mu_{\mathrm{F}}$. 

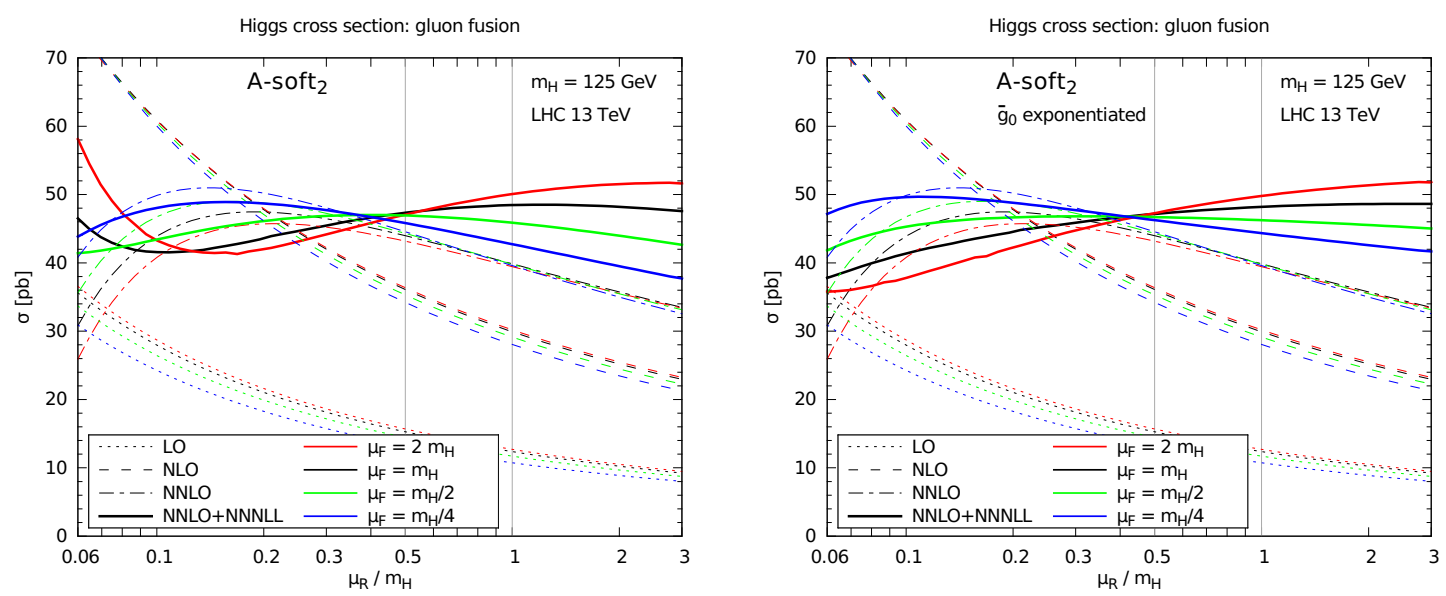

Figure 7. Same as figure 3 but for $\sqrt{s}=13 \mathrm{TeV}$.
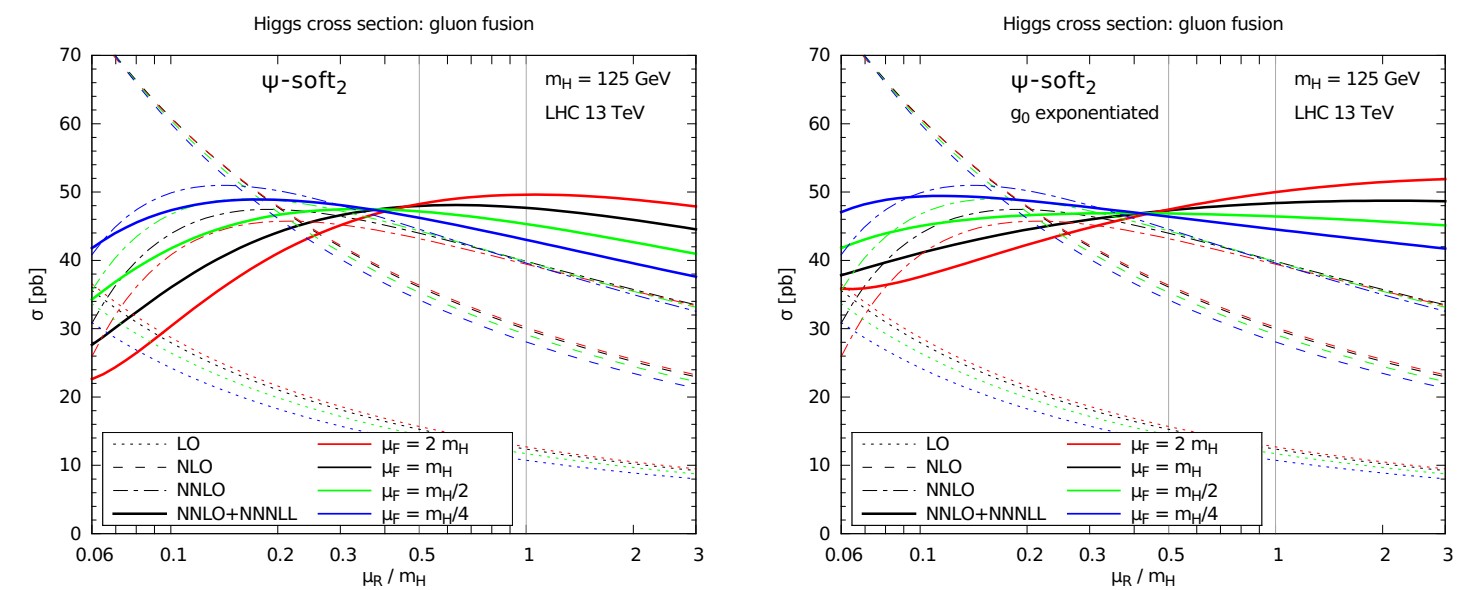

Figure 8. Same as figure 4 but for $\sqrt{s}=13 \mathrm{TeV}$.

A quantitative comparison between the different resummed results is shown in figure 6 , where ratios to our best prediction, namely $A$-soft 2 with the exponentiated constant $\bar{G}_{0}$, are plotted as a function of $\mu_{\mathrm{R}}$, for different choices of $\mu_{\mathrm{F}}$. As previously observed, we confirm here quantitatively that the result obtained with $\psi$-soft 2 with $g_{0}$ exponentiated (solid red line) is almost identical to our best prediction, the difference being always below $1 \%$, and confirming that this prescription can be indeed used as a numerically convenient alternative to $A$-soft 2 with $\bar{G}_{0}$. We also observe that for a wide choice of scales not exponentiating $\bar{g}_{0}$ in $A$-soft 2 (dashed black line) leads to a result which only differs from the result with $\bar{G}_{0}$ by a few percent. In contrast, the difference between resummed results with $g_{0}$, e.g. dotted red curve, or its exponentiated version $G_{0}$, e.g. solid red curve, is more pronounced. Finally, we note that the difference between our best prediction and $N$-soft is about $10 \%$ for $\mu_{\mathrm{R}}=\mu_{\mathrm{F}}=m_{\mathrm{H}}$. 


\begin{tabular}{|rcc|}
\hline \multicolumn{3}{|c|}{$\mu_{\mathrm{R}}=\mu_{\mathrm{F}}=m_{\mathrm{H}}$} \\
$\sqrt{s}$ & $\mathrm{NNLO}$ & $\mathrm{NNLO}+\mathrm{N}^{3} \mathrm{LL}$ \\
\hline $7 \mathrm{TeV}$ & $13.59_{-1.46}^{+1.64} \mathrm{pb}$ & $16.65_{-0.63}^{+1.42} \mathrm{pb}$ \\
$8 \mathrm{TeV}$ & $17.36_{-1.81}^{+2.06} \mathrm{pb}$ & $21.19_{-0.81}^{+1.81} \mathrm{pb}$ \\
$13 \mathrm{TeV}$ & $39.86_{-3.79}^{+4.45} \mathrm{pb}$ & $48.19_{-1.95}^{+4.15} \mathrm{pb}$ \\
$14 \mathrm{TeV}$ & $44.94_{-4.20}^{+4.94} \mathrm{pb}$ & $54.27_{-2.25}^{+4.70} \mathrm{pb}$ \\
$33 \mathrm{TeV}$ & $161.5_{-14.4}^{+16.2} \mathrm{pb}$ & $193.4_{-9.3}^{+17.2} \mathrm{pb}$ \\
\hline
\end{tabular}

\begin{tabular}{|ccc|}
\hline \multicolumn{3}{|c|}{$\mu_{\mathrm{R}}=\mu_{\mathrm{F}}=m_{\mathrm{H}} / 2$} \\
$\sqrt{s}$ & $\mathrm{NNLO}$ & $\mathrm{NNLO}+\mathrm{N}^{3} \mathrm{LL}$ \\
\hline $7 \mathrm{TeV}$ & $15.23_{-1.64}^{+1.62} \mathrm{pb}$ & $16.08_{-0.09}^{+0.57} \mathrm{pb}$ \\
$8 \mathrm{TeV}$ & $19.42_{-2.06}^{+2.07} \mathrm{pb}$ & $20.48_{-0.13}^{+0.71} \mathrm{pb}$ \\
$13 \mathrm{TeV}$ & $44.31_{-4.50}^{+4.81} \mathrm{pb}$ & $46.70_{-0.46}^{+1.52} \mathrm{pb}$ \\
$14 \mathrm{TeV}$ & $49.89_{-5.08}^{+5.47} \mathrm{pb}$ & $52.56_{-0.53}^{+1.81} \mathrm{pb}$ \\
$33 \mathrm{TeV}$ & $177.6_{-18.9}^{+20.0} \mathrm{pb}$ & $187.6_{-3.6}^{+7.6} \mathrm{pb}$ \\
\hline
\end{tabular}

Table 2. Values of the NNLO and NNLO+N ${ }^{3} \mathrm{LL}\left(A\right.$-soft ${ }_{2}$ with $\left.\bar{G}_{0}\right)$ gluon fusion cross section for selected values of the collider energy. We use NNPDF2.3 [65] with $m_{\mathrm{H}}=125 \mathrm{GeV}, m_{\mathrm{t}}=172.5 \mathrm{GeV}$, $m_{\mathrm{b}}=4.75 \mathrm{GeV}$ and $m_{\mathrm{c}}=1.41 \mathrm{GeV}$. The central value is for $\mu_{\mathrm{R}}=\mu_{\mathrm{F}}=m_{\mathrm{H}}$ (on the left) and $\mu_{\mathrm{R}}=\mu_{\mathrm{F}}=m_{\mathrm{H}} / 2$ (on the right). As detailed in the text, we recommend to evaluate theoretical uncertainties by scale variation around $\mu_{\mathrm{R}}=\mu_{\mathrm{F}}=m_{\mathrm{H}}$. Electro-weak corrections are not included.

In figures 7 and 8 , we show the results for $A$-soft 2 and $\psi$-soft 2 with collider energy $\sqrt{s}=13 \mathrm{TeV}$. The shape of the NNLO+N3 $\mathrm{LL}$ cross section in terms of renormalization and factorization scales, is very similar to the ones we have found at $8 \mathrm{TeV}$, both for $A$-soft 2 and $\psi$-soft 2 , and the analogous ratios showed in figure 6 for $8 \mathrm{TeV}$ look almost identical at $13 \mathrm{TeV}$. Thus, most of the points we have discussed for the $8 \mathrm{TeV}$ case, also apply at $13 \mathrm{TeV}$. We just note that the NLO cross section exhibits a somewhat larger $\mu_{\mathrm{F}}$ dependence at $13 \mathrm{TeV}$ than at $8 \mathrm{TeV}$.

\subsection{Numerical results for different collider energies}

In table 2 we summarise the results for our best prediction, namely $A$-soft s $_{2}$ with $\bar{G}_{0}$, for different collider energies and different values of the Higgs mass. We estimate the theoretical uncertainty by independently varying the scales up and down, by a factor of two $m_{\mathrm{x}} / 2<\mu_{\mathrm{R}}, \mu_{\mathrm{F}}<2 m_{\mathrm{x}}$, with the condition $1 / 2<\mu_{\mathrm{R}} / \mu_{\mathrm{F}}<2$, where $m_{\mathrm{x}}=m_{\mathrm{H}}$ (on the left) and $m_{\mathrm{x}}=m_{\mathrm{H}} / 2$ (on the right).

Several comments can be made on the results in table 2. We first discuss on the effect of the resummation with respect to NNLO. If $\mu_{\mathrm{R}}=\mu_{\mathrm{F}}=m_{\mathrm{H}}$ is chosen as the central scale, we find that the inclusion of the resummation increases the NNLO prediction by $22 \%$ at $\sqrt{s}=7,8 \mathrm{TeV}$ and $21 \%$ at $\sqrt{s}=13,14 \mathrm{TeV}$. This is consistent with the $16 \%$ increase we previously found just for $\mathrm{N}^{3} \mathrm{LO}_{\text {approx }}[37,38]$. We remind the reader that the absolute value of the cross sections reported in table 2 is lower than the one of our previous studies because we now include bottom and charm quarks in the loops. On the other hand, if $\mu_{\mathrm{R}}=\mu_{\mathrm{F}}=m_{\mathrm{H}} / 2$ is chosen as the central scale, we find that the resummation only corrects the NNLO result by roughly $5.5 \%$ at $\sqrt{s}=7,8 \mathrm{TeV}$ and $\sqrt{s}=13,14 \mathrm{TeV}$.

It is important to note that, while the NNLO cross sections at $\mu_{\mathrm{R}}=\mu_{\mathrm{F}}=m_{\mathrm{H}}$ and $\mu_{\mathrm{R}}=\mu_{\mathrm{F}}=m_{\mathrm{H}} / 2$ differ by more than $10 \%$, the NNLO $+\mathrm{N}^{3} \mathrm{LL}$ is much more stable and only varies by $3 \%$ (in the opposite direction with respect to the change of the fixed-order). Thus, as expected, the resummation of soft-enhanced contributions reduces the theoretical uncertainty related to the choice of the hard scale. 
However, while the NNLO $+\mathrm{N}^{3} \mathrm{LL}$ central values in table 2 are rather similar, their uncertainties differ significantly. The total uncertainty band ${ }^{5}$ at $\mu_{\mathrm{R}}=\mu_{\mathrm{F}}=m_{\mathrm{H}}$ is around $12-13 \%$, while at $\mu_{\mathrm{R}}=\mu_{\mathrm{F}}=m_{\mathrm{H}} / 2$ is $4-4.5 \%$. This dramatic reduction of the scale dependence can be understood thanks to the study we have performed in section 4.1, where we have noticed, for instance, that $A$-soft 2 (and $\psi$-soft 2 ) curves with $\mu_{\mathrm{F}}=m_{\mathrm{H}} / 2$ barely depend on $\mu_{\mathrm{R}}$. However, in section 4.1 we have also noticed that resummed curves for different $\mu_{\mathrm{F}}$ all meet to a point which is not too far away from $\mu_{\mathrm{R}}=m_{\mathrm{H}} / 2$, resulting into an artificially small scale dependence, which is probably not representative of the true theoretical uncertainty.

Therefore, in order not to underestimate the uncertainty of the NNLO $+\mathrm{N}^{3} \mathrm{LL}$ result, we recommend to vary the scales around $\mu_{\mathrm{R}}=\mu_{\mathrm{F}}=m_{\mathrm{H}}$, which results into a fairly conservative $12-13 \%$ uncertainty band, yet significantly smaller than the $20-22 \%$ band of the NNLO result. One can also imagine implementing less canonical scale choices. For instance, the plots in section 4.1 seem to suggest that varying the scales around $\mu_{\mathrm{F}}=m_{\mathrm{H}} / 2$ and $\mu_{\mathrm{R}}=m_{\mathrm{H}}$ would lead to a less conservative, but still reliable, estimate of the theoretical uncertainty.

We also compute the cross section for a future, very energetic run of the LHC, with $\sqrt{s}=33 \mathrm{TeV}^{6}$. The relations between fixed-order and resummed results, and their uncertainty bands, are qualitatively similar to the ones found at lower energies. However, we note that at $\sqrt{s}=33 \mathrm{TeV}$ we may be becoming sensitive to high-energy (BFKL) corrections, that we are not resumming here. These corrections have negligible impact on the inclusive Higgs cross section at current LHC energies, but can play a significant role for future high-energy runs.

Our analysis only includes QCD corrections. Electro-Weak contributions [70-74] can be also taken into account at NLO and if one assumes that they factorize from QCD corrections, they typically lead to a few percent increase of the cross section. Moreover, our calculations assume that the Higgs boson is produced on its mass shell. However, off-shell effects can be relevant for precision Higgs physics, even if the Higgs is light [75]. We leave the inclusion of these effects, as well as a study of PDF uncertainties, to future work.

Finally, it is interesting to compare our result to the lower-order prediction NNLO+NNLL obtained with $N$-soft, which is the accuracy of the QCD calculation of the cross section currently recommended by the Higgs Cross section Working Group [68, 69], for the central scale $\mu_{\mathrm{F}}=\mu_{\mathrm{R}}=m_{\mathrm{H}}$. At $8 \mathrm{TeV}$, for instance, the cross section we obtain ${ }^{7}$ at this accuracy is $18.57_{-1.81}^{+2.06} \mathrm{pb}$, with a total uncertainty band of $15 \%$. Hence, our calculation

\footnotetext{
${ }^{5}$ We quote the total uncertainty band, because the uncertainties we find are fairly asymmetric.

${ }^{6}$ Because of their slow numerical convergence, NNLO+N $\mathrm{N}^{3} \mathrm{LL}$ results for $\sqrt{s}=33 \mathrm{TeV}$ have been computed using $\psi$-soft 2 rather than the default $A$-soft 2 . We have checked that in all the other cases this choice leads to differences at the permille level.

${ }^{7}$ The number presented here slightly differs to the one in refs. [68, 69]. Most of the difference comes from the presence of Electro-Weak contributions in the result of ref. [42], which leads to an increase of about $5 \%$ [70-74]. The remaining difference (about 1\%) is due to the different PDF set used and the lack of finite top mass correction at NNLO in ref. [42].
} 
leads to a $14 \%$ increase with respect to what is currently used by the LHC experiments, and reduces the theory uncertainty.

\section{Conclusions}

Accurate theoretical predictions for Higgs production in gluon-gluon fusion are of primary importance for the LHC physics programme. In this paper we have studied the all-order resummation of enhanced contributions due to the emissions of soft gluons.

Our resummation ( $A$-soft 2 ) differs from the traditional one (called here $N$-soft), in that it is build in such a way to respect the analyticity properties of coefficient functions $[37,38]$. We improve on our previous work by computing all-order $\mathrm{N}^{3} \mathrm{LL}$ matched to NNLO cross sections for proton-proton collisions at different center-of-mass energies. Our result therefore accounts for all the contributions also present in the $\mathrm{N}^{3} \mathrm{LO}$ soft-virtual approximation of ref. [35]. Matching to full $\mathrm{N}^{3} \mathrm{LO}$, when this becomes available, will be straightforward.

Because of its analyticity properties, our results can be also easily matched to the resummation of high-energy (BFKL) contributions, which we leave for future work. However, high-energy resummation by itself has a relatively small direct numerical impact, at current LHC energies, so we do not expect this inclusion to significantly alter the results presented in this paper. This situation can change, if future, very high-energy colliders are considered.

We have also shown that the traditional resummation formula can be minimally modified $(\psi$-soft 2$)$ in such a way that the final result is almost identical to $A$-soft 2 , when constants are exponentiated in both cases. This provides a fast alternative to implement our improved soft-gluon resummation results.

The resummation presented here has been implemented in a code called ResHiggs which can be interfaced with the fixed-order code ggHiggs. Both codes are publicly available at the website http://www.ge.infn.it/ bonvini/higgs/.

We have shown that, at energies relevant for LHC Run-I and Run-II, the resummation $(A$-soft 2$)$ corrects the NNLO result by as much as $20 \%$ at $\mu_{\mathrm{R}}=\mu_{\mathrm{F}}=m_{\mathrm{H}}$, while the correction is much smaller, $5.5 \%$, at $\mu_{\mathrm{R}}=\mu_{\mathrm{F}}=m_{\mathrm{H}} / 2$. However, the central value of $\mathrm{NNLO}+\mathrm{N}^{3} \mathrm{LL}$ results depends very mildly on the scale choice. Moreover, the result obtained at $\mathrm{NNLO}+\mathrm{N}^{3} \mathrm{LL}(A$-soft 2 ) leads to a $14 \%$ increase with respect to the $N$-soft result at NNLO+NNLL [42] at $\mu_{\mathrm{R}}=\mu_{\mathrm{F}}=m_{\mathrm{H}}$, corresponding to the accuracy recommended by the Higgs Cross section Working Group [68, 69] and currently used by the LHC experiments. We have also argued that theoretical uncertainties are better estimated by scale variations about $\mu_{\mathrm{R}}=\mu_{\mathrm{F}}=m_{\mathrm{H}}$, with a resulting $12-13 \%$ total band.

Our results have a moderate dependence on the renormalization scale, which instead drives the theoretical uncertainty of fixed-order calculations. The dependence on the factorization scale instead is somewhat larger than in fixed-order calculations. This is mainly due to the fact that we only resum the $g g$ channel. This situation is likely to improve, if we were able to resum at least the leading logarithms in the $q g$ channel, which are of the form $\alpha_{s}^{k} \log ^{2 k-1}(1-z)$, using techniques similar to the ones developed in refs. [76, 77] for 
deep-inelastic scattering or by resumming the whole class of the so-called next-to-eikonal contributions [78-80].

\section{Acknowledgments}

We thank Richard Ball, Stefano Forte and Giovanni Ridolfi for many useful discussions, and Frank Tackmann for a critical reading of the manuscript. The work of SM is supported by the U.K.'s STFC.

\section{A Prescriptions and their implementations}

\section{A.1 Minimal Prescription}

The physical cross section, eq. (2.1), can be written in terms of the $N$-space coefficient function as

$$
\frac{\sigma\left(\tau, M^{2}\right)}{\tau \sigma_{0}\left(M^{2}\right)}=\frac{1}{2 \pi i} \int_{c-i \infty}^{c+i \infty} d N \tau^{-N} \mathscr{L}(N) C\left(N, \alpha_{s}\right),
$$

where we have suppressed the flavor indices. The parameter $c$ has to be larger than the real part of the rightmost singularity of the integrand. At the resummed level, the presence of the cut for real $N>N_{L}=\exp \frac{1}{2 \beta_{0} \alpha_{s}}$ makes it impossible to find such a value of $c$.

The Minimal Prescription consists in computing the integral eq. (A.1) at the resummed level by simply choosing $c$ to the left of the cut and to the right of all the other singularities; moreover, the integration contour is rotated counterclockwise on the upper plane $\operatorname{Im} N>0$ and clockwise on the lower plane $\operatorname{Im} N<0$ to guarantee numerical convergence [48]. We write

$$
\frac{\sigma\left(\tau, M^{2}\right)}{\tau \sigma_{0}\left(M^{2}\right)} \stackrel{\mathrm{MP}}{=} \frac{1}{2 \pi i} \int_{\mathrm{MPc}} d N \tau^{-N} \mathscr{L}(N) C_{\mathrm{res}}\left(N, \alpha_{s}\right),
$$

where by MPc we denote the contour described above. The integral in eq. (A.2) is finite, and it is proven to be an asymptotic sum of the divergent series of the order-by-order inverse Mellin transform of $C_{N \text {-soft }}$ [48]. However, because of the presence of the cut, the integration cannot be closed to the right, with the consequence that the result does not vanish for $\tau>1$. If one tries to interpret the result as a convolution of a parton luminosity and a partonic cross section, the latter does not vanish for $z>1$; this contribution from the unphysical region $z>1$ violates factorization, but it is exponentially suppressed in $\Lambda_{\mathrm{QCD}}[48]$.

From a practical point of view, the computation of the integral in eq. (A.2) requires the knowledge of the parton luminosity $\mathscr{L}(N)$ for values of $N$ along the contour. However, the Mellin transform

$$
\mathscr{L}(N)=\int_{0}^{1} d x x^{N-1} \mathscr{L}(x)
$$

converges numerically only for $\operatorname{Re} N>0$, while the MPc contour involves values of $N$ with negative real part. Therefore, for an efficient practical realization of the MP, the Mellin transform eq. (A.3) must be computed analytically, meaning that we need a functional form for $\mathscr{L}(x)$. 
A very efficient way of approximating $\mathscr{L}(x)$ is obtained expanding on a basis of Chebyshev polynomials the function

$$
f(u)=\left[\frac{x^{\beta}}{(1-x)^{\gamma}} \mathscr{L}(x)\right]_{x=e^{u}}
$$

on the range $u_{\min }<u<0$, with $u_{\min } \leq \log \tau$, and where $\beta$ and $\gamma$ are parameters aimed to make $f(u)$ as flat as possible (by default we use $\beta=1$ and $\gamma=0$ ). Fast routines are available for computing the coefficient of the expansion of $f(u)$ on a basis of Chebyshev polynomials. After straightforward manipulations [53] we are able to write, for integer $\gamma$,

$$
\mathscr{L}(x)=(1-x)^{\gamma} x^{-\beta} \sum_{k=0}^{n} c_{k} \log ^{k} x=\sum_{j=0}^{\gamma}\left(\begin{array}{l}
\gamma \\
j
\end{array}\right)(-1)^{j} x^{j-\beta} \sum_{k=0}^{n} c_{k} \log ^{k} x,
$$

where $n$ is the order of the Chebyshev approximation and $c_{k}$ are coefficients which depend on $\mu_{\mathrm{F}}$. Its Mellin transform is

$$
\mathscr{L}(N)=\sum_{j=0}^{\gamma}\left(\begin{array}{l}
\gamma \\
j
\end{array}\right) \sum_{k=0}^{n} c_{k} \frac{(-1)^{k+j} k !}{(N+j-\beta)^{k+1}}
$$

suggesting that a small value for $\gamma$ is advisable to reduce the number of terms in the sum. This luminosity can then be used in eq. (A.2) for numerical evaluation. The whole procedure leads to a very fast numerical implementation: therefore, where the MP is applicable, its usage is preferred.

\section{A.2 Borel Prescription}

A prescription that deals directly with the divergent nature of the series of the orderby-order Mellin inversion of $C_{N \text {-soft }}$ has been proposed in refs. [49-53]. This prescription adopts a Borel method for summing the divergent series, and it is therefore called Borel Prescription (BP). We briefly review here the derivation, while referring to the original works for a detailed discussion.

The $k$-th derivative of a function can be written as

$$
\begin{aligned}
f^{(k)}(0) & =\frac{k !}{2 \pi i} \oint \frac{d \xi}{\xi^{k+1}} f(\xi) \\
& =\frac{1}{2 \pi i} \int_{0}^{\infty} d w e^{-w} \oint \frac{d \xi}{\xi} f(\xi)\left(\frac{w}{\xi}\right)^{k},
\end{aligned}
$$

where in the first line we have used the Cauchy formula (the integration encloses the singular point $\xi=0$ ) and in the second line we have rewritten the $k$ ! as an integral. As a result, the $k$-th derivative of $f$ has been translated into an integral operator acting on the $k$-th power of the variable $w / \xi$.

This operator can be used to translate a power of $\log N$ in eq. (2.17) into

$$
\log ^{k} \frac{1}{N}=\frac{1}{2 \pi i} \int_{0}^{\infty} d w e^{-w} \oint \frac{d \xi}{\xi} N^{-\xi}\left(\frac{w}{\xi}\right)^{k}
$$


so that we can rewrite

$$
C_{N \text {-soft }}\left(N, \alpha_{s}\right)=\frac{1}{2 \pi i} \int_{0}^{\infty} d w e^{-w} \oint \frac{d \xi}{\xi} N^{-\xi} C_{N \text {-soft }}\left(e^{-w / \xi}, \alpha_{s}\right) .
$$

Notice that the function $C_{N \text {-soft }}\left(e^{-w / \xi}, \alpha_{s}\right)$ has a cut in $-2 \beta_{0} \alpha_{s} w<\xi<0$, due to the Landau pole. Therefore, the $\xi$ integration contour, which must encircle the cut, extends to minus infinity for $w \rightarrow \infty$, where the oscillatory behavior of the integrand makes the integral divergent. This shows that the series is not Borel summable. The Borel Prescription is now formulated as [49-53]

$$
C_{N \text {-soft }}\left(N, \alpha_{s}\right) \stackrel{\text { BP }}{=} \frac{1}{2 \pi i} \int_{0}^{\frac{W}{2 \beta_{0} \alpha_{s}}} d w e^{-w} \oint \frac{d \xi}{\xi} N^{-\xi} C_{N \text {-soft }}\left(e^{-w / \xi}, \alpha_{s}\right)
$$

where the $w$ integral has been cut off, and $W$ is minimally $W=2$ (corresponding to the inclusion of twist 4 terms). This cut-off makes the integral convergent, because the cut extends always on a finite range. Since the BP is applied directly to the coefficient function $C_{N \text {-soft }}$, the physical cross section maintain its convolution structure, without any violation of factorization.

As stressed already in section 2.3, the numerical result of eq. (A.10) is virtually identical to that obtained with the MP eq. (A.2), since for phenomenologically relevant kinematic configurations the series is behaving perturbatively and the way the divergence of the series is tamed is immaterial [53].

One of the advantages of the BP is that the inverse Mellin transform can be computed analytically, since the $N$ dependence is confined in the generating function $N^{-\xi}$. The convolution with the parton luminosity can hence be computed directly in $z$ space, without the need of any approximation (although in practice approximations might be convenient numerically, see ref. [53]). The price to pay is a slower implementation, since the BP consists of two integrals, while the MP needs just one integration.

Another advantage, which is the main reason to consider the $\mathrm{BP}$, is the fact that the form of the soft terms is completely under control. Indeed, in the derivation of eq. (2.17) from eq. (2.7), a large- $N$ limit of the Mellin transform of the soft terms eq. (2.15) is taken, in order to transform the complicated derivatives of $\Gamma$ functions into simple powers of $\log N$, so that a closed form for the functions $g_{i}\left(\alpha_{s} \log N\right)$ can be found. The BP makes possible to skip this large- $N$ limit, since the derivatives can be translated into powers through the operator eq. (A.7). This can be obtained by simply replacing the generating function $N^{-\xi}$ in eq. (A.10) with the generating function of the derivatives in eq. (3.2), which corresponds to introducing soft terms with the right analyticity properties (see discussion there).

Moreover, in deriving eq. (2.17) all constant terms from the Mellin transform of plus distributions have been (by choice) removed from the exponent and put into the function $g_{0}\left(\alpha_{s}\right)$, eq. (2.25). Here we have the opportunity to also restore these constants in the exponent, since they were there in the original expression eq. (2.7).

However, the proper way to restore the original logarithms and constants is to let the BP operator act directly on the exponent $\mathcal{S}\left(\alpha_{s}, \log N\right)$. Therefore, we propose a new 
version of the BP,

$$
C_{A \text {-soft }}\left(N, \alpha_{s}\right) \stackrel{\text { BP }}{=} \bar{g}_{0}\left(\alpha_{s}\right) \exp \left\{\frac{1}{2 \pi i} \int_{0}^{\frac{W}{2 \beta_{0} \alpha_{s}}} d w e^{-w} \oint \frac{d \xi}{\xi}\left[\frac{\Gamma(N-\xi / 2)}{\Gamma(N+\xi / 2)}-\frac{1}{\Gamma(1+\xi)}\right] \mathcal{S}\left(\alpha_{s},-\frac{w}{\xi}\right)\right\},
$$

which can be further improved by letting the Altarelli-Parisi operator, eq. (3.6), act on the generating function, as in eq. (3.9). Since the conversion of soft terms is done at the exponent, the two integrals of the $\mathrm{BP}$ have to be computed for each value of $N$, and then the inverse Mellin transform has to be computed numerically. In practice, we can use the same numerical technique used for the MP (an integral of the form eq. (A.2), with the luminosity approximated as eq. (A.6)), with the exception that now the function eq. (A.11) does no longer have a cut, and hence the inverse Mellin transform exists. The resulting numerical implementation is slow, compared to the MP. However, this procedure is, to our knowledge, the only viable solution to reproduce the structure of eq. (2.7) to all orders in a numerical way.

Open Access. This article is distributed under the terms of the Creative Commons Attribution License (CC-BY 4.0), which permits any use, distribution and reproduction in any medium, provided the original author(s) and source are credited.

\section{References}

[1] S. Catani, D. de Florian, M. Grazzini and P. Nason, Soft gluon resummation for Higgs boson production at hadron colliders, JHEP 07 (2003) 028 [hep-ph/0306211] [INSPIRE].

[2] M. Cacciari, M. Czakon, M. Mangano, A. Mitov and P. Nason, Top-pair production at hadron colliders with next-to-next-to-leading logarithmic soft-gluon resummation, Phys. Lett. B 710 (2012) 612 [arXiv:1111.5869] [INSPIRE]

[3] W. Beenakker, C. Borschensky, M. Krämer, A. Kulesza, E. Laenen et al., NNLL resummation for squark and gluino production at the LHC, arXiv:1404.3134 [INSPIRE].

[4] A. Djouadi, M. Spira and P.M. Zerwas, Production of Higgs bosons in proton colliders: QCD corrections, Phys. Lett. B 264 (1991) 440 [INSPIRE].

[5] S. Dawson, Radiative corrections to Higgs boson production, Nucl. Phys. B 359 (1991) 283 [INSPIRE].

[6] M. Spira, A. Djouadi, D. Graudenz and P.M. Zerwas, Higgs boson production at the LHC, Nucl. Phys. B 453 (1995) 17 [hep-ph/9504378] [INSPIRE].

[7] C. Anastasiou and K. Melnikov, Higgs boson production at hadron colliders in NNLO QCD, Nucl. Phys. B 646 (2002) 220 [hep-ph/0207004] [inSPIRE].

[8] R.V. Harlander and W.B. Kilgore, Next-to-next-to-leading order Higgs production at hadron colliders, Phys. Rev. Lett. 88 (2002) 201801 [hep-ph/0201206] [INSPIRE].

[9] V. Ravindran, J. Smith and W.L. van Neerven, NNLO corrections to the total cross-section for Higgs boson production in hadron hadron collisions, Nucl. Phys. B 665 (2003) 325 [hep-ph/0302135] [INSPIRE]. 
[10] S. Marzani, R.D. Ball, V. Del Duca, S. Forte and A. Vicini, Higgs production via gluon-gluon fusion with finite top mass beyond next-to-leading order, Nucl. Phys. B 800 (2008) 127 [arXiv:0801.2544] [INSPIRE].

[11] R.V. Harlander and K.J. Ozeren, Top mass effects in Higgs production at next-to-next-to-leading order QCD: Virtual corrections, Phys. Lett. B 679 (2009) 467 [arXiv:0907.2997] [INSPIRE].

[12] A. Pak, M. Rogal and M. Steinhauser, Virtual three-loop corrections to Higgs boson production in gluon fusion for finite top quark mass, Phys. Lett. B 679 (2009) 473 [arXiv: 0907.2998] [INSPIRE].

[13] A. Pak, M. Rogal and M. Steinhauser, Finite top quark mass effects in NNLO Higgs boson production at LHC, JHEP 02 (2010) 025 [arXiv:0911.4662] [INSPIRE].

[14] R.V. Harlander and K.J. Ozeren, Finite top mass effects for hadronic Higgs production at next-to-next-to-leading order, JHEP 11 (2009) 088 [arXiv:0909.3420] [INSPIRE].

[15] R.V. Harlander, H. Mantler, S. Marzani and K.J. Ozeren, Higgs production in gluon fusion at next-to-next-to-leading order QCD for finite top mass, Eur. Phys. J. C 66 (2010) 359 [arXiv: 0912.2104] [INSPIRE].

[16] P.A. Baikov, K.G. Chetyrkin, A.V. Smirnov, V.A. Smirnov and M. Steinhauser, Quark and gluon form factors to three loops, Phys. Rev. Lett. 102 (2009) 212002 [arXiv:0902.3519] [INSPIRE].

[17] T. Gehrmann, E.W.N. Glover, T. Huber, N. Ikizlerli and C. Studerus, Calculation of the quark and gluon form factors to three loops in QCD, JHEP 06 (2010) 094 [arXiv: 1004.3653] [INSPIRE].

[18] C. Anastasiou, C. Duhr, F. Dulat and B. Mistlberger, Soft triple-real radiation for Higgs production at N3LO, JHEP 07 (2013) 003 [arXiv: 1302.4379] [INSPIRE].

[19] T. Gehrmann, M. Jaquier, E.W.N. Glover and A. Koukoutsakis, Two-Loop QCD Corrections to the Helicity Amplitudes for $H \rightarrow 3$ partons, JHEP 02 (2012) 056 [arXiv:1112.3554] [INSPIRE].

[20] C. Duhr and T. Gehrmann, The two-loop soft current in dimensional regularization, Phys. Lett. B 727 (2013) 452 [arXiv: 1309.4393] [INSPIRE].

[21] Y. Li and H.X. Zhu, Single soft gluon emission at two loops, JHEP 11 (2013) 080 [arXiv: 1309.4391] [INSPIRE].

[22] C. Anastasiou, C. Duhr, F. Dulat, F. Herzog and B. Mistlberger, Real-virtual contributions to the inclusive Higgs cross-section at $N^{3} L O$, JHEP 12 (2013) 088 [arXiv:1311.1425] [INSPIRE].

[23] W.B. Kilgore, One-Loop Single-Real-Emission Contributions to $p p \rightarrow H+X$ at Next-to-Next-to-Next-to-Leading Order, Phys. Rev. D 89 (2014) 073008 [arXiv:1312.1296] [INSPIRE].

[24] C. Anastasiou, S. Buehler, C. Duhr and F. Herzog, NNLO phase space master integrals for two-to-one inclusive cross sections in dimensional regularization, JHEP 11 (2012) 062 [arXiv: 1208.3130] [INSPIRE].

[25] M. Höschele, J. Hoff, A. Pak, M. Steinhauser and T. Ueda, Higgs boson production at the LHC: NNLO partonic cross sections through order $\epsilon$ and convolutions with splitting functions to $N^{3} L O$, Phys. Lett. B 721 (2013) 244 [arXiv:1211.6559] [INSPIRE]. 
[26] S. Buehler and A. Lazopoulos, Scale dependence and collinear subtraction terms for Higgs production in gluon fusion at N3LO, JHEP 10 (2013) 096 [arXiv:1306.2223] [INSPIRE].

[27] Y. Li, A. von Manteuffel, R.M. Schabinger and H.X. Zhu, $N^{3}$ LO Higgs and Drell-Yan production at threshold: the one-loop two-emission contribution, arXiv:1404.5839 [INSPIRE].

[28] K.G. Chetyrkin, B.A. Kniehl and M. Steinhauser, Decoupling relations to $O\left(\alpha_{S}^{3}\right)$ and their connection to low-energy theorems, Nucl. Phys. B 510 (1998) 61 [hep-ph/9708255] [INSPIRE].

[29] Y. Schröder and M. Steinhauser, Four-loop decoupling relations for the strong coupling, JHEP 01 (2006) 051 [hep-ph/0512058] [INSPIRE].

[30] K.G. Chetyrkin, J.H. Kuhn and C. Sturm, QCD decoupling at four loops, Nucl. Phys. B 744 (2006) 121 [hep-ph/0512060] [inSPIRE].

[31] O.V. Tarasov, A.A. Vladimirov and A.Y. Zharkov, The Gell-Mann-Low Function of QCD in the Three Loop Approximation, Phys. Lett. B 93 (1980) 429 [InSPIRE].

[32] S.A. Larin and J.A.M. Vermaseren, The Three loop QCD $\beta$-function and anomalous dimensions, Phys. Lett. B 303 (1993) 334 [hep-ph/9302208] [INSPIRE].

[33] T. van Ritbergen, J.A.M. Vermaseren and S.A. Larin, The Four loop $\beta$-function in quantum chromodynamics, Phys. Lett. B 400 (1997) 379 [hep-ph/9701390] [INSPIRE].

[34] M. Czakon, The Four-loop QCD $\beta$-function and anomalous dimensions, Nucl. Phys. B 710 (2005) 485 [hep-ph/0411261] [INSPIRE].

[35] C. Anastasiou, C. Duhr, F. Dulat, E. Furlan, T. Gehrmann et al., Higgs boson gluon-fusion production at threshold in N3LO QCD, arXiv:1403.4616 [INSPIRE].

[36] M. Bonvini, S. Forte and G. Ridolfi, The Threshold region for Higgs production in gluon fusion, Phys. Rev. Lett. 109 (2012) 102002 [arXiv:1204.5473] [INSPIRE].

[37] R.D. Ball, M. Bonvini, S. Forte, S. Marzani and G. Ridolfi, Higgs production in gluon fusion beyond NNLO, Nucl. Phys. B 874 (2013) 746 [arXiv:1303.3590] [INSPIRE].

[38] M. Bonvini, R.D. Ball, S. Forte, S. Marzani and G. Ridolfi, Updated Higgs cross section at approximate $N^{3} L O, J$. Phys. G 41 (2014) 095002 [arXiv:1404.3204] [INSPIRE].

[39] S. Catani and L. Trentadue, Resummation of the QCD Perturbative Series for Hard Processes, Nucl. Phys. B 327 (1989) 323 [inSPIRE].

[40] G.F. Sterman, Summation of Large Corrections to Short Distance Hadronic Cross-Sections, Nucl. Phys. B 281 (1987) 310 [inSPIRE].

[41] S. Forte and G. Ridolfi, Renormalization group approach to soft gluon resummation, Nucl. Phys. B 650 (2003) 229 [hep-ph/0209154] [INSPIRE].

[42] D. de Florian and M. Grazzini, Higgs production at the LHC: updated cross sections at $\sqrt{s}=8$ TeV, Phys. Lett. B 718 (2012) 117 [arXiv:1206.4133] [InSPIRE].

[43] V. Ahrens, T. Becher, M. Neubert and L.L. Yang, Renormalization-Group Improved Prediction for Higgs Production at Hadron Colliders, Eur. Phys. J. C 62 (2009) 333 [arXiv: 0809.4283] [INSPIRE].

[44] M. Bonvini, S. Forte, M. Ghezzi and G. Ridolfi, The scale of soft resummation in SCET vs perturbative QCD, Nucl. Phys. Proc. Suppl. 241-242 (2013) 121 [arXiv:1301.4502] [INSPIRE]. 
[45] S. Moch, J.A.M. Vermaseren and A. Vogt, Higher-order corrections in threshold resummation, Nucl. Phys. B 726 (2005) 317 [hep-ph/0506288] [INSPIRE].

[46] S. Moch and A. Vogt, Higher-order soft corrections to lepton pair and Higgs boson production, Phys. Lett. B 631 (2005) 48 [hep-ph/0508265] [INSPIRE].

[47] E. Laenen and L. Magnea, Threshold resummation for electroweak annihilation from DIS data, Phys. Lett. B 632 (2006) 270 [hep-ph/0508284] [INSPIRE].

[48] S. Catani, M.L. Mangano, P. Nason and L. Trentadue, The Resummation of soft gluons in hadronic collisions, Nucl. Phys. B 478 (1996) 273 [hep-ph/9604351] [INSPIRE].

[49] S. Forte, G. Ridolfi, J. Rojo and M. Ubiali, Borel resummation of soft gluon radiation and higher twists, Phys. Lett. B 635 (2006) 313 [hep-ph/0601048] [INSPIRE].

[50] R. Abbate, S. Forte and G. Ridolfi, A New prescription for soft gluon resummation, Phys. Lett. B 657 (2007) 55 [arXiv:0707.2452] [INSPIRE].

[51] M. Bonvini, S. Forte and G. Ridolfi, Borel resummation of transverse momentum distributions, Nucl. Phys. B 808 (2009) 347 [arXiv:0807.3830] [INSPIRE].

[52] M. Bonvini, S. Forte and G. Ridolfi, Soft gluon resummation of Drell-Yan rapidity distributions: Theory and phenomenology, Nucl. Phys. B 847 (2011) 93 [arXiv:1009.5691] [INSPIRE].

[53] M. Bonvini, Resummation of soft and hard gluon radiation in perturbative QCD, arXiv: 1212.0480 [INSPIRE].

[54] M. Krämer, E. Laenen and M. Spira, Soft gluon radiation in Higgs boson production at the LHC, Nucl. Phys. B 511 (1998) 523 [hep-ph/9611272] [INSPIRE].

[55] H. Contopanagos, E. Laenen and G.F. Sterman, Sudakov factorization and resummation, Nucl. Phys. B 484 (1997) 303 [hep-ph/9604313] [INSPIRE].

[56] S. Catani, D. de Florian and M. Grazzini, Higgs production in hadron collisions: Soft and virtual QCD corrections at NNLO, JHEP 05 (2001) 025 [hep-ph/0102227] [INSPIRE].

[57] G. Parisi, Summing Large Perturbative Corrections in QCD, Phys. Lett. B 90 (1980) 295 [INSPIRE].

[58] L. Magnea and G.F. Sterman, Analytic continuation of the Sudakov form-factor in QCD, Phys. Rev. D 42 (1990) 4222 [inSPIRE].

[59] A.P. Bakulev, A.V. Radyushkin and N.G. Stefanis, Form-factors and QCD in space - like and time - like region, Phys. Rev. D 62 (2000) 113001 [hep-ph/0005085] [INSPIRE].

[60] D.J. Broadhurst, A.L. Kataev and C.J. Maxwell, Renormalons and multiloop estimates in scalar correlators: Higgs decay and quark mass sum rules, Nucl. Phys. B 592 (2001) 247 [hep-ph/0007152] [INSPIRE].

[61] V. Ahrens, T. Becher, M. Neubert and L.L. Yang, Origin of the Large Perturbative Corrections to Higgs Production at Hadron Colliders, Phys. Rev. D 79 (2009) 033013 [arXiv: 0808.3008] [INSPIRE].

[62] I.W. Stewart, F.J. Tackmann, J.R. Walsh and S. Zuberi, Jet $p_{T}$ Resummation in Higgs Production at NNLL' + NNLO, Phys. Rev. D 89 (2014) 054001 [arXiv:1307.1808] [INSPIRE]. 
[63] T.O. Eynck, E. Laenen and L. Magnea, Exponentiation of the Drell-Yan cross-section near partonic threshold in the DIS and MS-bar schemes, JHEP 06 (2003) 057 [hep-ph/0305179] [INSPIRE].

[64] R. Bonciani, G. Degrassi and A. Vicini, Scalar particle contribution to Higgs production via gluon fusion at NLO, JHEP 11 (2007) 095 [arXiv: 0709.4227] [INSPIRE].

[65] R.D. Ball, V. Bertone, S. Carrazza, C.S. Deans, L. Del Debbio et al., Parton distributions with LHC data, Nucl. Phys. B 867 (2013) 244 [arXiv:1207.1303] [INSPIRE].

[66] S. Forte, A. Isgrò and G. Vita, Do we need $N^{3} L O$ Parton Distributions?, Phys. Lett. B 731 (2014) 136 [arXiv:1312.6688] [INSPIRE].

[67] C. Anastasiou, S. Buehler, F. Herzog and A. Lazopoulos, Inclusive Higgs boson cross-section for the LHC at $8 \mathrm{TeV}$, JHEP 04 (2012) 004 [arXiv:1202.3638] [INSPIRE].

[68] LHC Higgs Cross Section Working Group collaboration, S. Dittmaier et al., Handbook of LHC Higgs Cross Sections: 1. Inclusive Observables, arXiv:1101.0593 [INSPIRE].

[69] https://twiki.cern.ch/twiki/bin/view/LHCPhysics/LHCHXSWG.

[70] G. Degrassi and F. Maltoni, Two-loop electroweak corrections to Higgs production at hadron colliders, Phys. Lett. B 600 (2004) 255 [hep-ph/0407249] [INSPIRE].

[71] U. Aglietti, R. Bonciani, G. Degrassi and A. Vicini, Two-loop electroweak corrections to Higgs production in proton-proton collisions, hep-ph/0610033 [INSPIRE].

[72] S. Actis, G. Passarino, C. Sturm and S. Uccirati, NLO Electroweak Corrections to Higgs Boson Production at Hadron Colliders, Phys. Lett. B 670 (2008) 12 [arXiv:0809.1301] [INSPIRE].

[73] S. Actis, G. Passarino, C. Sturm and S. Uccirati, NNLO Computational Techniques: The Cases $H \rightarrow \gamma \gamma$ and $H \rightarrow g g$, Nucl. Phys. B 811 (2009) 182 [arXiv:0809.3667] [InSPIRE].

[74] C. Anastasiou, R. Boughezal and F. Petriello, Mixed QCD-electroweak corrections to Higgs boson production in gluon fusion, JHEP 04 (2009) 003 [arXiv: 0811.3458] [INSPIRE].

[75] N. Kauer and G. Passarino, Inadequacy of zero-width approximation for a light Higgs boson signal, JHEP 08 (2012) 116 [arXiv:1206.4803] [INSPIRE].

[76] A. Vogt, Leading logarithmic large-x resummation of off-diagonal splitting functions and coefficient functions, Phys. Lett. B 691 (2010) 77 [arXiv:1005.1606] [INSPIRE].

[77] A.A. Almasy, G. Soar and A. Vogt, Generalized double-logarithmic large-x resummation in inclusive deep-inelastic scattering, JHEP 03 (2011) 030 [arXiv:1012.3352] [INSPIRE].

[78] E. Laenen, L. Magnea, G. Stavenga and C.D. White, Next-to-eikonal corrections to soft gluon radiation: a diagrammatic approach, JHEP 01 (2011) 141 [arXiv:1010.1860] [INSPIRE].

[79] E. Laenen, G. Stavenga and C.D. White, Path integral approach to eikonal and next-to-eikonal exponentiation, JHEP 03 (2009) 054 [arXiv: 0811.2067] [INSPIRE].

[80] E. Laenen, L. Magnea and G. Stavenga, On next-to-eikonal corrections to threshold resummation for the Drell-Yan and DIS cross sections, Phys. Lett. B 669 (2008) 173 [arXiv: 0807.4412] [INSPIRE]. 